\title{
Charged Higgs bosons in naturally aligned two-Higgs-doublet models at the LHC
}

\author{
Emily Hanson, ${ }^{1}$ William Klemm, ${ }^{1}$ Roger Naranjo, ${ }^{2}$ Yvonne Peters, ${ }^{1}$ and Apostolos Pilaftsis ${ }^{1}$ \\ ${ }^{1}$ School of Physics and Astronomy, University of Manchester, Manchester M13 9PL, United Kingdom \\ ${ }^{2}$ Deutsches Elektronen-Synchrotron DESY, Hamburg 22607, Germany
}

(Received 10 January 2019; published 27 August 2019)

\begin{abstract}
Measurements of a Higgs boson at the Large Hadron Collider (LHC) have become increasingly consistent with the predictions of the Standard Model (SM). This fact puts severe constraints on many potential low-energy extensions of the Higgs sector of the SM. In the well-known two-Higgsdoublet model (2HDM), an "alignment limit" of parameters readily furnishes one SM-like scalar and can be achieved naturally through an underlying symmetry. Among the other physical states of the 2HDM, a charged scalar $H^{ \pm}$would provide striking evidence of new physics if observed. We propose a novel technique for the observation of the process $p p \rightarrow t b H^{ \pm} \rightarrow t \bar{t} b \bar{b}$ in the dileptonic decay channel at the LHC. The reconstruction of events in this channel is complicated by multiple $b$ jets and unobserved neutrinos in the final state. To determine the neutrino momenta, we implement a neutrino weighting procedure to study, for the first time, the $t \bar{t} b \bar{b}$ signature. We further train a pair of boosted decision trees to reconstruct and classify signal events. We determine the resulting reach within the context of naturally aligned 2HDMs, such as the maximally symmetric two-Higgs-doublet model (MS2HDM). By testing at the integrated luminosity of $150 \mathrm{fb}^{-1}$ achieved in run 2 of the LHC, we find that this channel may restrict the parameter space of a type-II MS2HDM with charged Higgs masses as high as $680 \mathrm{GeV}$.
\end{abstract}

DOI: $10.1103 /$ PhysRevD.100.035026

\section{INTRODUCTION}

One of the great achievements of the Large Hadron Collider (LHC) has been the discovery of a resonance around $125 \mathrm{GeV}[1,2]$, whose measured signal rates in dominant decay channels increasingly agree with that of a Standard Model (SM) Higgs boson [3]. The observation of a Higgs boson further opens the door for the possibility of extended Higgs sectors, with parameters constrained by measured properties. One of the simplest such extensions is the two-Higgs-doublet model (2HDM) [4], which introduces one additional electroweak isodoublet. Versions of the 2HDM appear in a variety of well-motivated scenarios for new physics, both with and without supersymmetry [5-7], in which the additional Higgs field is either an essential ingredient or necessary by-product in addressing issues such as the origin of dark matter, the generation of a baryon asymmetry, the gauge hierarchy problem, and the strong $C P$ problem.

Published by the American Physical Society under the terms of the Creative Commons Attribution 4.0 International license. Further distribution of this work must maintain attribution to the author(s) and the published article's title, journal citation, and DOI. Funded by SCOAP ${ }^{3}$.
Any version of the $2 \mathrm{HDM}$ attempting to describe the observed $125 \mathrm{GeV}$ state, $h$, must be able to reproduce the SM-like signals seen at the LHC. One simple way to achieve this, known as the "decoupling limit," is to set the masses of additional scalars so high that they play a minimal role around the electroweak scale [8]. Another possibility, which can lead to new scalars at energies accessible to the LHC, is the "alignment limit," where the parameters of the theory force one $C P$-even scalar to have SM-like couplings [9-15]. While this limit can be achieved by pure conspiracy of parameters, it is more natural to consider the possibility that it arises from an underlying symmetry $[10,14,16-18]$. The simplest scenario, dubbed the maximally symmetric two-Higgsdoublet model (MS2HDM), has been shown to be a viable option with new states accessible at LHC energies $[10,18]$.

One possible striking signature of naturally aligned 2HDMs comes from the existence of a charged scalar state $H^{ \pm}$, present in some extended Higgs sectors and general 2HDMs. Collider searches to date have yielded constraints on models containing a charged Higgs, but the 2HDM parameter space still contains unexplored regions which could be accessible with continued running of the LHC $[19,20]$. The decays of a sufficiently heavy charged Higgs 
boson are typically dominated by $H^{ \pm} \rightarrow t b$, giving the possible $p p \rightarrow t b H^{ \pm} \rightarrow t \bar{t} b \bar{b}$ signature. ${ }^{1}$ As the top quarks can decay either hadronically or leptonically, there are a few possible resulting final states, each of which poses its own challenges for reconstruction and classification. Here we focus on the dileptonic channel, where both top quarks decay to a $b$ jet, charged lepton, and neutrino.

This article is organized as follows. In Sec. II we review the 2HDM and the naturally aligned MS2HDM. In Sec. III we introduce a novel analysis for identifying a charged Higgs boson at the LHC in the dileptonic decay channel and determine the resulting reach for an $H^{ \pm}$in the MS2HDM. Finally, the results of our analysis are summarized in Sec. IV.

\section{THE TWO-HIGGS-DOUBLET MODEL}

The two complex scalar Higgs fields, transforming as isodoublets $(2,1)$ under the SM electroweak gauge group $\mathrm{SU}(2)_{L} \otimes \mathrm{U}(1)_{Y}$, may be represented as

$$
\Phi_{i}=\left(\begin{array}{c}
\phi_{i}^{+} \\
\phi_{i}^{0}
\end{array}\right)
$$

with $i=1,2$; then the most general $2 \mathrm{HDM}$ potential may be written as

$$
\begin{aligned}
V= & -\mu_{1}^{2}\left(\Phi_{1}^{\dagger} \Phi_{1}\right)-\mu_{2}^{2}\left(\Phi_{2}^{\dagger} \Phi_{2}\right)-\left[m_{12}^{2}\left(\Phi_{1}^{\dagger} \Phi_{2}\right)+\text { H.c. }\right] \\
& +\lambda_{1}\left(\Phi_{1}^{\dagger} \Phi_{1}\right)^{2}+\lambda_{2}\left(\Phi_{2}^{\dagger} \Phi_{2}\right)^{2}+\lambda_{3}\left(\Phi_{1}^{\dagger} \Phi_{1}\right)\left(\Phi_{2}^{\dagger} \Phi_{2}\right) \\
& +\lambda_{4}\left(\Phi_{1}^{\dagger} \Phi_{2}\right)\left(\Phi_{2}^{\dagger} \Phi_{1}\right) \\
& +\left[\frac{1}{2} \lambda_{5}\left(\Phi_{1}^{\dagger} \Phi_{2}\right)^{2}+\lambda_{6}\left(\Phi_{1}^{\dagger} \Phi_{1}\right)\left(\Phi_{1}^{\dagger} \Phi_{2}\right)\right. \\
& \left.+\lambda_{7}\left(\Phi_{1}^{\dagger} \Phi_{2}\right)\left(\Phi_{2}^{\dagger} \Phi_{2}\right)+\text { H.c. }\right] .
\end{aligned}
$$

This contains four real mass parameters $\mu_{1,2}^{2}, \operatorname{Re}\left(m_{12}^{2}\right)$, $\operatorname{Im}\left(m_{12}^{2}\right)$, and ten real quartic couplings $\lambda_{1,2,3,4}, \operatorname{Re}\left(\lambda_{5,6,7}\right)$, and $\operatorname{Im}\left(\lambda_{5,6,7}\right)$. Of these 14 parameters, three parameters can be removed by a $U(2)$ reparameterization of the Higgs doublets $\Phi_{1}$ and $\Phi_{2}$ [21]. If we assume $C P$ conservation, which allows the SM-like Higgs to be a $C P$-even scalar, then the parameters in (2) are required to be real. After electroweak symmetry breaking, each isodoublet acquires a vacuum expectation value (VEV) $v_{j}$ such that $\sqrt{v_{1}^{2}+v_{2}^{2}}=$ $v \approx 246 \mathrm{GeV}$ and $\phi_{j}^{0}=\left(v_{j}+\phi_{j}+i a_{j}\right) / \sqrt{2}$, where $\phi_{j}$ and $a_{j}$ are real scalar fields. Three degrees of freedom become the longitudinal modes of the electroweak gauge bosons, leaving five physical states: two $C P$-even scalars $h, H$ with

\footnotetext{
${ }^{1}$ In this work, we consider both $H^{+}$and $H^{-}$together; quark and antiquark assignments may be inferred. Thus, in our notation, $\sigma\left(p p \rightarrow t b H^{ \pm}\right)=\sigma\left(p p \rightarrow t \bar{b} H^{-}\right)+\sigma\left(p p \rightarrow \bar{t} b H^{+}\right)$.
}

$m_{h}<m_{H}$; one $C P$-odd pseudoscalar $A$; and two charged scalars $H^{ \pm}$. It is then often useful to reexpress the mass parameters $\mu_{1,2}^{2}$ and quartic couplings $\lambda_{1}-\lambda_{5}$ in terms of the physical masses $m_{h}, m_{H}, m_{A}$, and $m_{H^{ \pm}}$, along with the ratio of VEVs, $\tan \beta=v_{2} / v_{1}$ and the neutral sector mixing term $\sin (\beta-\alpha)$. The angles $\alpha$ and $\beta$ govern the mixing between mass eigenstates in the $C P$-even sector and $C P$-odd and charged sectors, respectively.

Each Higgs field has Yukawa interactions with SM fermions, with the quark-sector Yukawa Lagrangian given by

$-\mathcal{L}_{Y}^{q}=\bar{Q}_{L}\left(h_{1}^{u} \tilde{\Phi}_{1}+h_{2}^{u} \tilde{\Phi}_{2}\right) u_{R}+\bar{Q}_{L}\left(h_{1}^{d} \Phi_{1}+h_{2}^{d} \Phi_{2}\right) d_{R}$,

where $Q_{L}=\left(u_{L}, d_{L}\right)^{T}$ is the $S U(2)_{L}$ quark doublet, $u_{R}$ and $d_{R}$ are right-handed quark singlets, and $\tilde{\Phi}_{i}=i \sigma_{2} \Phi_{i}^{*}$ are the isospin conjugates of $\Phi_{i}$. A similar expression holds for the leptons. Because the potential contains couplings which mix the two isodoublets, a general $2 \mathrm{HDM}$ will produce tree-level flavor-changing neutral currents (FCNCs). One way to suppress these FCNCs is to impose the GlashowWeinberg condition [22], introducing a discrete $Z_{2}$ symmetry under which charges are assigned to ensure that each type of fermion couples to only a single Higgs doublet. If the fields transform as

$\Phi_{1} \rightarrow-\Phi_{1}, \quad \Phi_{2} \rightarrow \Phi_{2}, \quad u_{R} \rightarrow u_{R}, \quad d_{R} \rightarrow \pm d_{R}$,

then at tree level the up-type quarks acquire mass solely from $\Phi_{2}$ and the down-type quarks acquire mass solely from $\Phi_{2}(+)$ or from $\Phi_{1}(-)$. Including the leptons, there are four possible unique assignments; here we will focus mainly on the type-II $2 \mathrm{HDM}$, in which $\Phi_{2}$ couples only to up-type quarks and $\Phi_{1}$ couples to down-type quarks and charged leptons, as in the minimal supersymmetric standard model.

\section{A. Natural alignment in the 2HDM}

The couplings of $h$ and $H$ to SM gauge bosons are related to the SM value by a factor of $\sin (\beta-\alpha)$ and $\cos (\beta-\alpha)$, respectively, ${ }^{2}$ such that when $\sin (\beta-\alpha)=1$ $(0), h(H)$ has SM-like gauge couplings. In addition, the couplings of fermions to the neutral scalars are related to the SM value for $h$ by $\cos \alpha / \sin \beta$ or $-\sin \alpha / \cos \beta$, depending on the type of fermion and $Z_{2}$ symmetry, and by $\sin \alpha / \sin \beta$ or $\cos \alpha / \cos \beta$ for $H$. Therefore, when $\sin (\beta-\alpha)=1(0), h(H)$ also has SM-like couplings to fermions. When this alignment condition is met, one of the neutral scalars looks identical to a SM Higgs boson in its tree-level interactions with other SM particles.

\footnotetext{
${ }^{2} \mathrm{~A}$ different convention for $\alpha$ is sometimes chosen such that these assignments are reversed.
} 
The $C P$-even mass matrix in the $2 \mathrm{HDM}$ may be expressed as [10]

$$
M_{S}^{2}=\left(\begin{array}{cc}
c_{\beta} & -s_{\beta} \\
s_{\beta} & c_{\beta}
\end{array}\right)\left(\begin{array}{cc}
\hat{A} & \hat{C} \\
\hat{C} & \hat{B}
\end{array}\right)\left(\begin{array}{cc}
c_{\beta} & s_{\beta} \\
-s_{\beta} & c_{\beta}
\end{array}\right),
$$

where $c_{\beta}=\cos \beta, s_{\beta}=\sin \beta$, and

$$
\begin{aligned}
& \hat{A}=2 v^{2}\left[c_{\beta}^{4} \lambda_{1}+s_{\beta}^{2} c_{\beta}^{2} \lambda_{345}+s_{\beta}^{4} \lambda_{2}+2 s_{\beta} c_{\beta}\left(c_{\beta}^{2} \lambda_{6}+s_{\beta}^{2} \lambda_{7}\right)\right], \\
& \hat{B}= m_{A}^{2}+\lambda_{5} v^{2}+2 v^{2}\left[s_{\beta}^{2} c_{\beta}^{2}\left(\lambda_{1}+\lambda_{2}-\lambda_{345}\right)\right. \\
&\left.-s_{\beta} c_{\beta}\left(c_{\beta}^{2}-s_{\beta}^{2}\right)\left(\lambda_{6}-\lambda_{7}\right)\right], \\
& \hat{C}= v^{2}\left[s_{\beta}^{3} c_{\beta}\left(2 \lambda_{2}-\lambda_{345}\right)-c_{\beta}^{3} s_{\beta}\left(2 \lambda_{1}-\lambda_{345}\right)\right. \\
&\left.+c_{\beta}^{2}\left(1-4 s_{\beta}^{2}\right) \lambda_{6}+s_{\beta}^{2}\left(4 c_{\beta}^{2}-1\right) \lambda_{7}\right],
\end{aligned}
$$

where $\lambda_{345}=\lambda_{3}+\lambda_{4}+\lambda_{5}$. The pseudoscalar mass $m_{A}$ and charged Higgs mass $m_{H^{ \pm}}$are, respectively,

$$
\begin{gathered}
m_{A}^{2}=m_{H^{ \pm}}^{2}+\frac{v^{2}}{2}\left(\lambda_{4}-\lambda_{5}\right), \\
m_{H^{ \pm}}=\frac{m_{12}^{2}}{s_{\beta} c_{\beta}}-\frac{v^{2}}{2}\left(\lambda_{4}+\lambda_{5}\right)+\frac{v^{2}}{2 s_{\beta} c_{\beta}}\left(\lambda_{6} c_{\beta}^{2}+\lambda_{7} s_{\beta}^{2}\right) .
\end{gathered}
$$

Diagonalization of $M_{S}^{2}$ gives the $C P$-even mass eigenstates $H$ and $h$ :

$$
\left(\begin{array}{c}
H \\
h
\end{array}\right)=\left(\begin{array}{cc}
c_{\alpha} & s_{\alpha} \\
-s_{\alpha} & c_{\alpha}
\end{array}\right)\left(\begin{array}{l}
\phi_{1} \\
\phi_{2}
\end{array}\right)
$$

such that

$$
\begin{aligned}
\left(\begin{array}{cc}
m_{H}^{2} & 0 \\
0 & m_{h}^{2}
\end{array}\right) & =\left(\begin{array}{cc}
c_{\alpha} & s_{\alpha} \\
-s_{\alpha} & c_{\alpha}
\end{array}\right) M_{S}^{2}\left(\begin{array}{cc}
c_{\alpha} & -s_{\alpha} \\
s_{\alpha} & c_{\alpha}
\end{array}\right) \\
& =\left(\begin{array}{cc}
c_{\beta-\alpha} & -s_{\beta-\alpha} \\
s_{\beta-\alpha} & c_{\beta-\alpha}
\end{array}\right)\left(\begin{array}{cc}
\hat{A} & \hat{C} \\
\hat{C} & \hat{B}
\end{array}\right)\left(\begin{array}{cc}
c_{\beta-\alpha} & s_{\beta-\alpha} \\
-s_{\beta-\alpha} & c_{\beta-\alpha}
\end{array}\right) .
\end{aligned}
$$

For the alignment condition, $\sin (\beta-\alpha)=1$ [or $\cos (\beta-\alpha)=$ 1], (12) may only be satisfied if $\hat{C}=0$. The alignment condition in a general $C P$-conserving $2 \mathrm{HDM}$ may then be expressed as

$$
\begin{aligned}
& \lambda_{7} t_{\beta}^{4}-\left(2 \lambda_{2}-\lambda_{345}\right) t_{\beta}^{3}+3\left(\lambda_{6}-\lambda_{7}\right) t_{\beta}^{2}+\left(2 \lambda_{1}-\lambda_{345}\right) t_{\beta}-\lambda_{6} \\
& \quad=0
\end{aligned}
$$

where $t_{\beta}=\tan \beta$ (see also [9] for an equivalent expression). To satisfy (13) for all values of $\tan \beta$, the coefficients of each power of $\tan \beta$ must vanish, thus yielding the conditions for natural alignment $[10,14]$ :

$$
\lambda_{1}=\lambda_{2}=\lambda_{345} / 2, \quad \lambda_{6}=\lambda_{7}=0 .
$$

The $U(1)_{Y}$-invariant 2 HDM potential contains 13 accidental symmetries, which have been fully classified in $[23,24]$ upon extending the bilinear field formalism in [25-27]. . $^{3}$ of these, three restrict the quartic couplings such that the natural alignment conditions of (14) are met [10,14]:

$$
\begin{aligned}
& S O(5): \lambda_{1}=\lambda_{2}=\lambda_{3} / 2, \quad \lambda_{4}=\lambda_{5}=\lambda_{6}=\lambda_{7}=0, \\
& O(3) \times O(2): \lambda_{1}=\lambda_{2}, \quad \lambda_{3}=2 \lambda_{1}-\lambda_{4}, \quad \lambda_{5}=\lambda_{6}=\lambda_{7}=0,
\end{aligned}
$$

$$
Z_{2} \times[O(2)]^{2}: \lambda_{1}=\lambda_{2}, \quad \lambda_{3}=2 \lambda_{1}-\left(\lambda_{4}+\lambda_{5}\right), \quad \lambda_{6}=\lambda_{7}=0
$$

While all the above symmetries are exactly realized when $\mu_{1}^{2}=\mu_{2}^{2}$ and $m_{12}^{2}=0$, their soft breaking by an arbitrary choice of the parameters $\mu_{1,2}^{2}$ and $m_{12}^{2}$ will still be sufficient to give rise to alignment, at least at the tree level. For this reason, we call (15)-(17) symmetries of natural alignment.

In the following, we will focus on the MS2HDM [10] which possesses an $S O(5)$ invariant potential in the extended bilinear field formalism [23]. The parameters in (15) produce one massive $C P$-even scalar with $m_{h}=2 \lambda_{1} v^{2}$. The other four physical scalars $\left(H, A, H^{ \pm}\right)$ become massless and would participate in decays of SM particles, which is inconsistent with observation. The custodial $S O(5)$ symmetry, which is violated by $U(1)_{Y}$ hypercharge and the Yukawa couplings, could be realized at some high scale $\mu_{X}$, with the electroweak scale behavior determined by the renormalization group (RG) evolution of the parameters, but this alone is unable to sufficiently raise the masses [10]. However, a viable Higgs spectrum is achievable by introducing a soft-breaking term $\operatorname{Re}\left(m_{12}^{2}\right)$, which yields

$$
m_{h}^{2}=2 \lambda_{1} v^{2}, \quad m_{H}^{2}=m_{A}^{2}=m_{H^{ \pm}}^{2}=\frac{\operatorname{Re}\left(m_{12}^{2}\right)}{s_{\beta} c_{\beta}} .
$$

As stated above, the alignment conditions in (14) do not depend on the soft-breaking parameters, e.g., $m_{12}^{2}$, and as such alignment will still occur at the symmetry-breaking scale. RG evolution to the electroweak scale will introduce some misalignment, but for a wide range of $\tan \beta$ and $\mu_{X}$, a viable low-energy theory is possible [10]. In the remainder of this work, we do not choose a particular scale $\mu_{X}$ to evaluate the RG evolution of parameters but define 2HDM parameters according to (15) and (18), ignoring RG effects. For the purposes of this study, this consideration provides

\footnotetext{
${ }^{3}$ We note that only six symmetries of 13 are preserved by $U(1)_{Y}$ gauge interactions beyond the tree-level approximation $[26,28,29]$.
} 

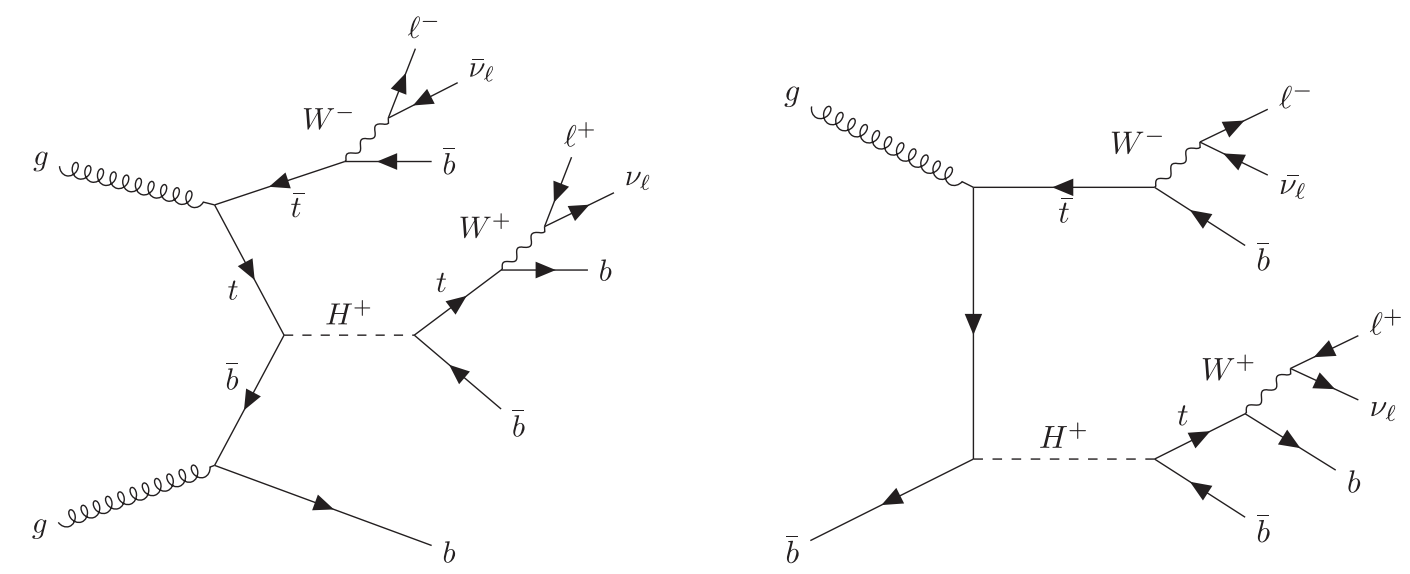

FIG. 1. Dileptonic decay channel of charged Higgs production in association with top quarks in the four-flavor and five-flavor schemes, respectively.

an appropriate working hypothesis for our numerical analysis that follows in Sec. III.

\section{B. Charged Higgs bosons in the 2HDM}

In the 2HDM, charged Higgs bosons have couplings to fermions given by

$$
\begin{aligned}
\mathcal{L}_{H^{ \pm}} & =-H^{+}\left(\frac{\sqrt{2} V_{u d}}{v} \bar{u}\left(m_{u} X P_{L}+m_{d} Y\right) d+\frac{\sqrt{2} m_{\ell}}{v} Z \bar{\nu}_{L} \ell_{R}\right) \\
& + \text { H.c. }
\end{aligned}
$$

where terms containing $u, d$, and $\ell$ are summed over three generations and $V_{u d}$ is the Cabibbo-Kobayashi-Maskawa matrix. In type-II models, the real parameters become $X=$ $\cot \beta$ and $Y=Z=-\tan \beta$. Because the couplings are proportional to fermion masses, the $H^{ \pm} t b$ coupling typically dominates; in type-II models, it is maximized at large and small $\tan \beta$. Consequently, this coupling can play a major role in charged Higgs production. A light charged Higgs can be produced through the top quark decay $t \rightarrow H^{ \pm} b$, and a heavy charged Higgs can be produced as $g g \rightarrow t b H^{ \pm}$, or in the five-flavor scheme, $g b \rightarrow t H^{ \pm}$, as seen in Fig. 1.

Charged Higgs bosons $H^{ \pm}$may also decay through their couplings to fermions, with a preference for heavier fermions when kinematically allowed. Numerous searches have been performed at LEP [30], Tevatron [31-33], and the LHC [34-38] for the decays of $H^{+}$to $\tau^{+} \nu_{\tau}, c \bar{s}$, and for sufficiently heavy $H^{+}$, to $t \bar{b}$. Charged Higgs bosons can, in principle, also decay to $W^{ \pm}$bosons and any of the neutral Higgs bosons, $h, H$ and $A$. Although $H^{ \pm} \rightarrow W^{ \pm} h$ can be observed by taking advantage of the already-measured properties of the observed $h$ [39], the $H^{ \pm} W^{\mp} h$ coupling is proportional to $\cos (\beta-\alpha)$, which vanishes in the alignment limit considered here. Moreover, in the MS2HDM, the near degeneracy of $H, A$, and $H^{ \pm}$leads to a kinematic suppression of the decays $H^{ \pm} \rightarrow W^{ \pm} H$ or $H^{ \pm} \rightarrow W^{ \pm} A$.
Then $p p \rightarrow t b H^{ \pm} \rightarrow t \bar{t} b \bar{b}$ is a natural search channel for a heavy $H^{ \pm}$in the MS2HDM. The ATLAS Collaboration has recently published such a search using $36.1 \mathrm{fb}^{-1}$ of data at $\sqrt{s}=13 \mathrm{TeV}$, combining dileptonic and semileptonic final states to place limits on $\sigma\left(p p \rightarrow t b H^{ \pm}\right) \times \mathrm{BR}\left(H^{ \pm} \rightarrow t b\right)$ ranging from $2.9 \mathrm{pb}$ at $m_{H^{ \pm}}=200 \mathrm{GeV}$ to $0.070 \mathrm{pb}$ at $m_{H^{ \pm}}=2000 \mathrm{GeV}$ [38].

The predicted signal cross sections for the production of heavy Higgs bosons in association with top quarks in the MS2HDM are shown in Figs. 2 and 3 for the four- and fiveflavor schemes, respectively. Like the charged Higgs bosons, the neutral Higgs bosons couple preferentially to third-generation fermions. This means that they can also mediate large $t \bar{t} b \bar{b}$ signals, also illustrated in Figs. 2 and 3. However, for additional Higgs boson masses which are nearly degenerate, charged Higgs production dominates this channel for a large range of $\tan \beta$.

In addition to direct searches, charged Higgs bosons can enhance rare flavor-changing decays, in particular, the $B$ meson decay $B \rightarrow X_{s} \gamma$. Precise measurement of these decay rates and calculation of their predicted values in the SM place tight constraints on type-II models, with lower limits on $m_{H^{ \pm}}$in the $570-800 \mathrm{GeV}$ range, with a high sensitivity to the exact method used [41]. Performing direct searches for $H^{ \pm}$at the LHC provides a complementary means of testing these limits while probing a larger mass range.

\section{CHARGED HIGGS SIGNAL AT THE LHC}

In this section, we consider the LHC production and decay of a charged Higgs boson via the process $p p \rightarrow$ $t(b) H^{ \pm} \rightarrow t \bar{t} b \bar{b}$, focusing on the dileptonic decay channel ${ }^{4}$ $\left(b b \bar{b} \bar{b} \ell^{+} \ell^{-} \nu_{\ell} \bar{\nu}_{\ell}\right)$, as illustrated in Fig. 1. The backgrounds we consider for this channel are $t \bar{t} b \bar{b}, t \bar{t} c \bar{c}$, and

\footnotetext{
${ }^{4}$ See [42] for a recent proposal for the hadronic and semileptonic channels.
} 

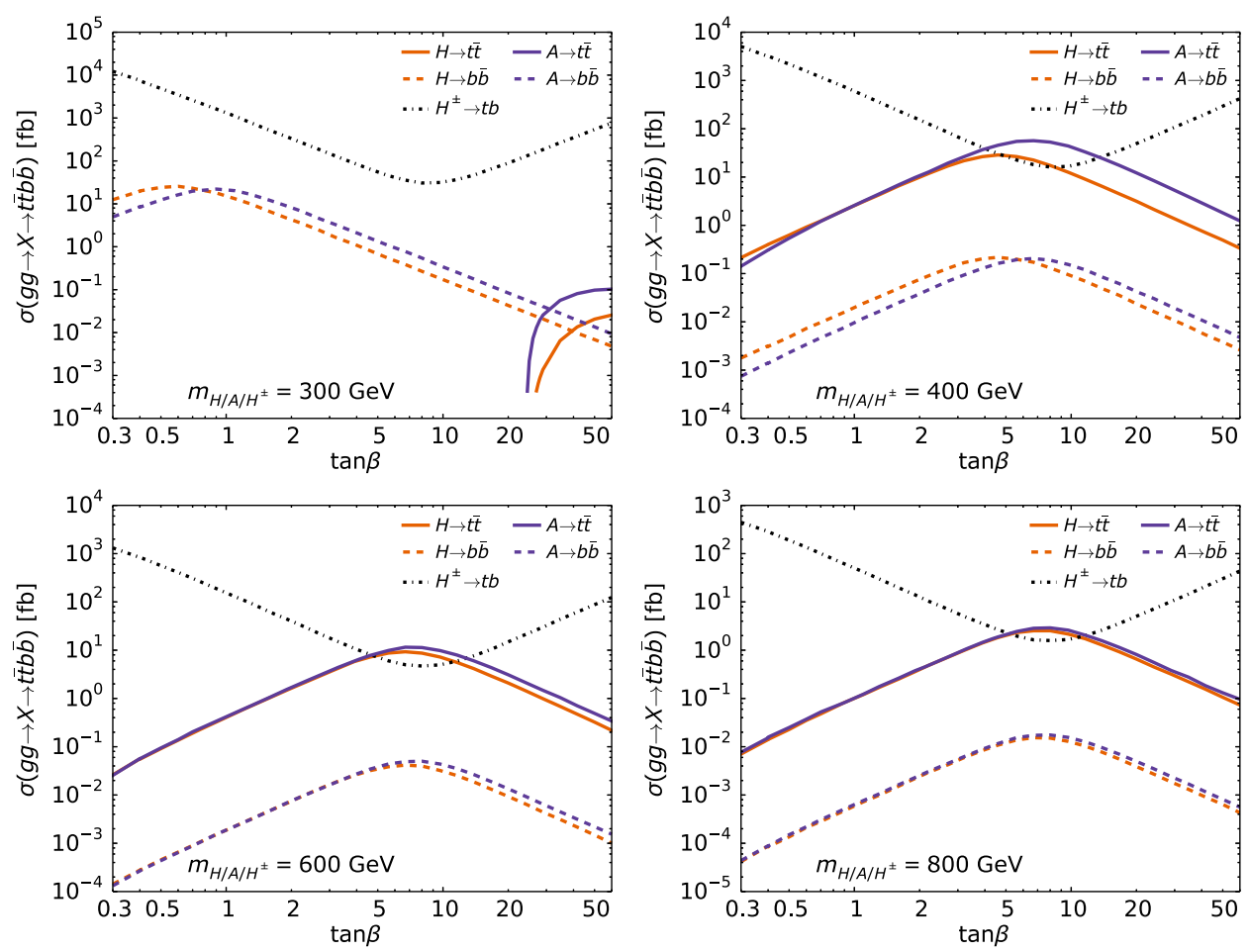

FIG. 2. Cross sections for $t \bar{t} b \bar{b}$ production in the MS2HDM in $p p$ collisions at $\sqrt{s}=13 \mathrm{TeV}$. The legend denotes how the heavy Higgs bosons decay. All cross sections here are calculated in the four-flavor scheme with no kinematic cuts with MADGRAPH5_AMC@NLO [40].
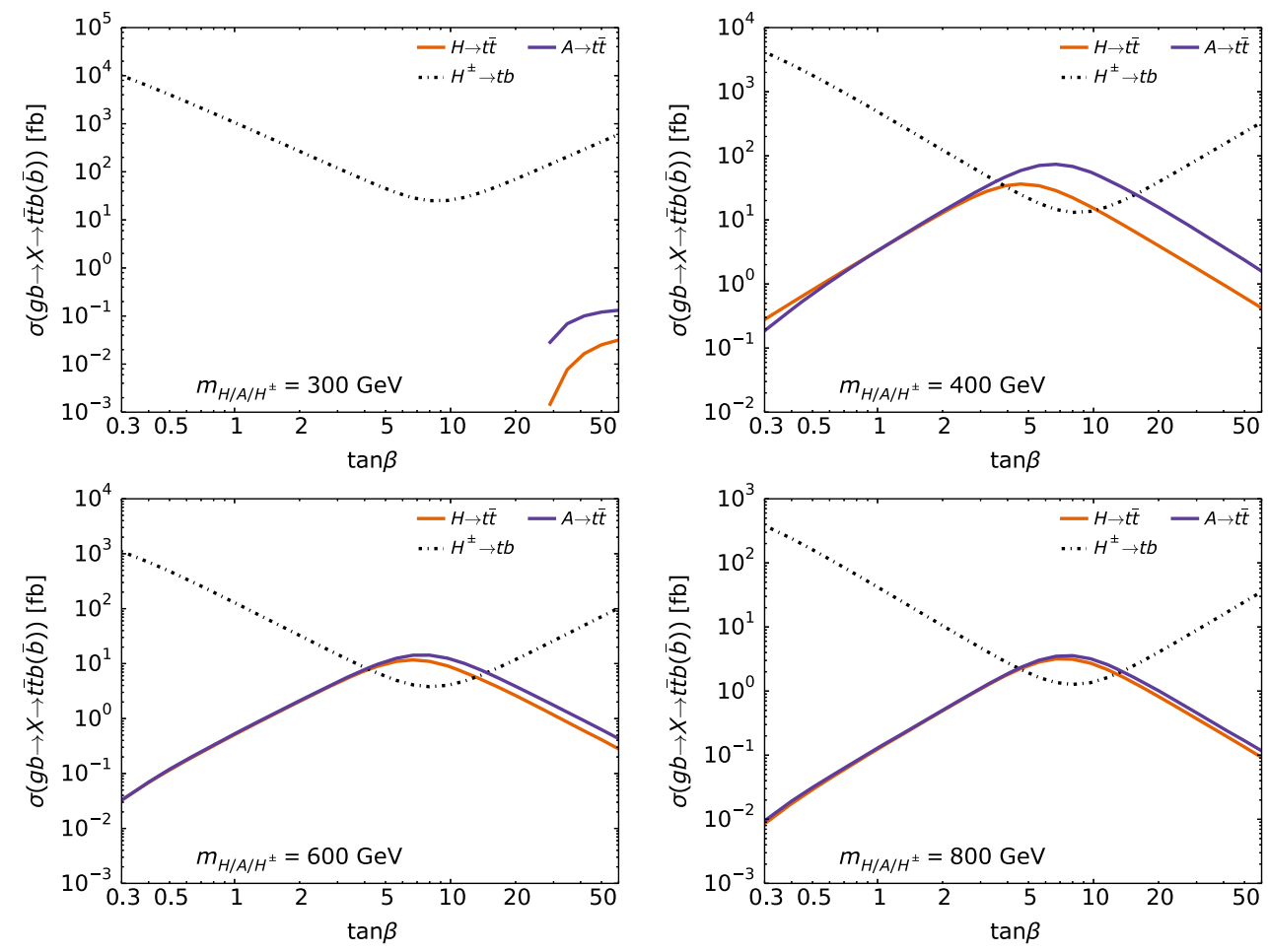

FIG. 3. The same as in Fig. 2, but in the five-flavor scheme. 
$t \bar{t}+$ light jets $(g, u, d, s)$. We generate signal and background events with MADGRAPH5_AMC@NLO [40], shower with Pythia6 [43], and finally perform jet reconstruction and detector simulation using FASTJET [44] and DELPHES3.4.1 [45], using the ATLAS configuration card. Jets are defined using the anti- $k_{t}$ algorithm with radius parameter $R=0.4$. The signal process for a type-II $2 \mathrm{HDM}$ in the alignment limit is generated using the 2HDMC [46] model for MAdGRAPH. For the signal, $t H^{ \pm}, t H^{ \pm} j$, and $t H^{ \pm} b$ samples are generated in the five-flavor scheme and matched using the MLM procedure [47], named after the first author, as implemented by MADGRAPH and PYTHIA, with a matching scale of $m_{H^{ \pm}} / 4$. The cross sections are then normalized to the Santander-matched cross sections given by the LHC Higgs Cross Section Working Group [48-52]. For the backgrounds, $t \bar{t}+0,1,2$ jet (five-flavor) samples are generated ${ }^{5}$ and matched at a scale of $80 \mathrm{GeV}$. Other minor backgrounds, such as singletop production, are not considered; these are shown to affect the final result by, at most, 3\%-4.5\%, and more commonly by less than $1 \%$. Interferences between the signal and background are also found to be negligible. Though other scalars in the 2HDM could contribute to a $t \bar{t} b \bar{b}$ signal, we restrict ourselves here to processes containing a charged Higgs boson. We generate samples of signal events with $\tan \beta=1,2,5,10,15,30,60$ and $200 \mathrm{GeV} \leq m_{H^{ \pm}} \leq 1000 \mathrm{GeV}$ in steps of $100 \mathrm{GeV}$. Additional samples with $\left(\tan \beta, m_{H^{ \pm}}\right)=(50,200)$, (40, $300),(50,300),(40,400),(50,400),(50,500)$ for finer granularity in the regions with most sensitivity.

In the dileptonic channel, the final state contains four $b$ quarks, two charged leptons $\left(\ell^{ \pm}=e^{ \pm}, \mu^{ \pm}\right)$, and two neutrinos, on which we impose an initial selection:

(i) Exactly two leptons with transverse momenta $p_{T} \geq 20 \mathrm{GeV}$, pseudorapidity $|\eta|<2.5$, invariant dilepton mass $m_{\ell \ell}>12 \mathrm{GeV}$ and $\left|m_{\ell \ell}-m_{Z}\right|>$ $10 \mathrm{GeV}$, with separation $\Delta R_{\ell \ell}>0.4$.

(ii) Missing transverse energy $\mathbb{E}_{T} \geq 40 \mathrm{GeV}$.

(iii) The event must contain at least three jets with $p_{T} \geq 25 \mathrm{GeV}$ and $|\eta|<2.4$, with a leading jet $p_{T} \geq 30 \mathrm{GeV}$; at least two of these jets must be $b$ tagged. A $b$-tagged jet is one that is identified as likely to contain a $b$ hadron. The sample is split into three-jet and $\geq 4$-jet regions. After ordering by $p_{T}$, the first four (three for three-jet events) $b$-tagged jets are taken if possible. If less than four jets are $b$ tagged, the highest $p_{T}$ non- $b$-tagged jet(s) are additionally taken to select four (three for three-jet events) total jets. These are henceforth collectively called $b$ jets.

The effect of each of these requirements is shown in Fig. 4 for an illustrative value of $\tan \beta=10$ and at three

\footnotetext{
${ }^{5}$ For practical reasons, samples are produced separately for different jet flavors accompanying the $t \bar{t}$ pair.
}

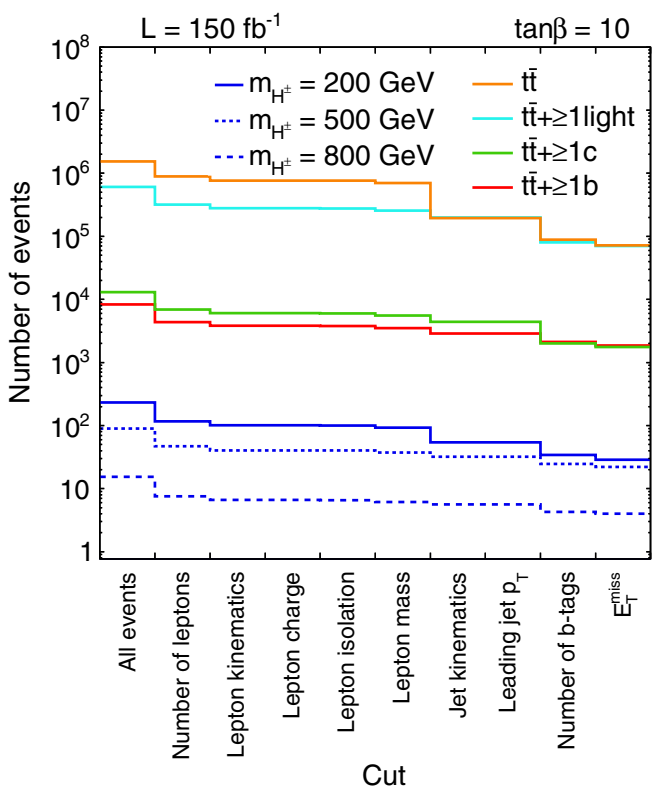

FIG. 4. The number of events, normalized to an integrated luminosity of $150 \mathrm{fb}^{-1}$, that survive after each stage of the selection process, for three signal masses at $\tan \beta=10$, overlaid with the SM backgrounds.

masses, $m_{H^{ \pm}}=200,500,800 \mathrm{GeV}$. The proportion of signal events that remain after selection is approximately constant across $\tan \beta$ for a given mass. The effect of the selection on the SM backgrounds is also shown in Fig. 4.

The reconstruction and classification of signal events in this channel present several challenges, which we address with a three-step analysis utilizing boosted decision trees (BDTs):

(i) a reconstruction BDT to identify the $b$ jets originating from the $b$ quarks $b_{t}, b_{t H}$, and $b_{H}$ as defined in Fig. 1,

(ii) a neutrino weighting procedure to reconstruct the two neutrino momenta and to identify the correct $b-\ell^{ \pm}$pairings in top decays, and

(iii) a classification BDT trained to distinguish signal and background events using the reconstruction from the first two steps. A template fit is performed on the output of this BDT to obtain limits.

\section{A. Boosted decision trees}

A BDT is a structure for classifying events by considering observables to produce a single value quantifying how signal- or backgroundlike a given event is. This is done by training on Monte Carlo samples of signal and background. A decision tree consists of several successive layers of nodes, beginning with a single root node. At each node, the variable providing the greatest discriminating power is determined, using the training events, and an appropriate cut is applied. This causes a split into two new nodes, one expected to contain the signal and one for the 
TABLE I. Performance of reconstruction BDT and neutrino weighting procedure for $\tan \beta=1-60$, with minimum and maximum values.

\begin{tabular}{|c|c|c|c|c|c|}
\hline & \multicolumn{5}{|c|}{$m_{H^{ \pm}}$} \\
\hline & $200 \mathrm{GeV}$ & $300 \mathrm{GeV}$ & $500 \mathrm{GeV}$ & $700 \mathrm{GeV}$ & $900 \mathrm{GeV}$ \\
\hline$b$ jet to quark matching [\%] & $34.51-38.86$ & $61.77-65.31$ & $60.04-65.25$ & $59.57-65.02$ & $58.80-64.70$ \\
\hline Reconstruction BDT separation & $0.62-0.69$ & $0.30-0.39$ & $0.52-0.60$ & $0.63-0.70$ & $0.70-0.75$ \\
\hline$\left(b_{t}, b_{H}, b_{t H}\right)$ correct $[\%]$ & $23.4-25.6$ & $19.4-24.2$ & $29.8-31.0$ & $34.2-36.7$ & $37.0-40.5$ \\
\hline Neutrino weighting solution exists [\%] & $90.1-91.9$ & $95.4-97.3$ & $93.3-95.9$ & $90.9-94.2$ & $88.3-93.3$ \\
\hline$H^{ \pm}$charge correct $[\%]$ (all events) & $55.9-58.6$ & $56.3-59.7$ & $58.3-59.4$ & $61.8-63.1$ & $65.2-66.4$ \\
\hline $\begin{array}{c}H^{ \pm} \text {charge correct }[\%] \text { [events } \\
\left.\text { with correct }\left(b_{t}, b_{H}, b_{t H}\right)\right]\end{array}$ & $84.0-85.8$ & $80.3-81.3$ & $82.3-84.1$ & $86.4-87.3$ & $88.8-89.3$ \\
\hline $\begin{array}{l}\left(b_{t}, b_{H}, b_{t H}\right) \text { correct and } H^{ \pm} \\
\quad \text { charge correct }[\%]\end{array}$ & $19.8-21.9$ & $15.6-19.7$ & $24.7-26.1$ & $29.8-31.8$ & $33.1-36.2$ \\
\hline
\end{tabular}

background, and the best discriminating variables for the new nodes are determined. This processing of splitting and creating new nodes continues until a newly created node receives a subset of training events, which contains less than a threshold number of events, meets a condition on purity (e.g., mostly signal events), or reaches a maximum tree depth. This node is designated as an end node which assigns a classification corresponding to the dominant type of event in the subset of training events it received. After training, each event can thus be categorized as "signal-like" or "backgroundlike."

Boosting is a procedure which combines several weak classifiers into a stronger classifier. When applied to decision trees, boosting has been shown to improve both performance and stability [53]. Once an initial decision tree has been generated, events in the training sample are assigned weights. Training events which are misclassified by the initial decision tree are weighted more heavily than those which are correctly classified. This new reweighted sample is then used to train a new decision tree, which may then be used to generate a new set of weights for the training sample to generate yet another decision tree. This procedure repeats several times to create a set of decision trees (a "forest"); when analyzing an event, each tree is queried for a classification (e.g., -1 for background, +1 for signal), and a weighted average of the responses gives a final score. Several different boosting algorithms exist with different weighting procedures for the training events and trees. In this work, we use the AdaBoost algorithm [54,55] with $\beta=0.5$ for the reconstruction $\mathrm{BDT}$ and the GradientBoost algorithm with shrinkage $=0.3$ for the classification BDT.

Throughout this work we implement BDTs using the TMVA package [53] to generate forests of 400 (100) trees for the reconstruction (classification) BDT, each with a maximum depth of three layers. The cuts at each node are chosen to minimize the sum of the Gini indices of the resulting subsets of events, weighted by the fraction of events in each subset, where Gini $=p(1-p)$ for a sample with signal purity $p=N_{\text {signal }} / N_{\text {total }}$.

\section{B. Reconstruction BDT}

As shown in Fig. 1, there are three $b$ jets whose origin must be determined: $b_{t}$ from the decay of the associated top, $b_{H}$ from the charged Higgs decay, and $b_{t H}$ from the top quark from the charged Higgs decay. An additional jet, $b_{g}$, is emitted from the initial gluon (or, in the five-flavor scheme, produced in the parton shower) and is not considered in the reconstruction BDT. The $b$ jets are matched to parton-level (truth) $b$ quarks by determining which jet-quark pairs have the smallest separation in $\eta-\phi$, called $\Delta R$, providing $\Delta R \leq 0.4$. The performance of this $b$ jet to quark matching procedure is shown in the first row of Table I. This shows that the matching is generally stable with mass, except at $200 \mathrm{GeV}$ where the efficiency is lower.

From the $b$ jet to quark matching, we know the true origin of each $b$ jet. Then, we iterate through all combinations of $b$ jets, labeling them $b_{t H}, b_{t}$ and $b_{H}$. A permutation is, therefore, correct if all three $b$ jets have the same true origin as the label assigned to them; otherwise, it is incorrect. In order to separate the correct permutation from all of the possible incorrect permutations for a given event, we train a reconstruction BDT on each of the signal samples. This takes advantage of variations in kinematics due to model parameters. In this BDT, the correct permutation in an event serves as the "signal," and all incorrect permutations are "background." For events where the matching procedure does not find pairings to all of $b_{t H}, b_{t}$, and $b_{H}$, all permutations in that event are background. The reconstruction BDT is trained on 57 observables:

(i) $\Delta R\left(b_{i}, l^{a}\right), \Delta \eta\left(b_{i}, l^{a}\right), \Delta \phi\left(b_{i}, l^{a}\right), p_{T}^{b_{i}+l^{a}}, m\left(b_{i}, l^{a}\right)$, where $i=t H, t$ and $a=+,-$;

(ii) $\left|m_{b_{j}}\left(l^{+}, b_{t H}\right)-m\left(l^{-}, b_{t}\right)\right|$ and $\left|m\left(l^{-}, b_{t H}\right)-m\left(l^{+}, b_{t}\right)\right|$;

(iii) $p_{T}^{b_{j}}$, where $j=t H, H, t$;

(iv) $\Delta R\left(b_{t H}, b_{k}\right), \Delta \eta\left(b_{t H}, b_{k}\right), \Delta \phi\left(b_{t H}, b_{k}\right), p_{T}^{b_{t H}+b_{k}}$, $m\left(b_{t H}, b_{k}\right)$, where $k=H, t$;

(v) $\Delta R\left(t_{H^{a}}, b_{H}\right), \Delta \eta\left(t_{H^{a}}, b_{H}\right), \Delta \phi\left(t_{H^{a}}, b_{H}\right), p_{T}^{t_{H^{a}}, b_{H}}$, $m\left(t_{H^{a}}, b_{H}\right)$, where $a=+,-$; 

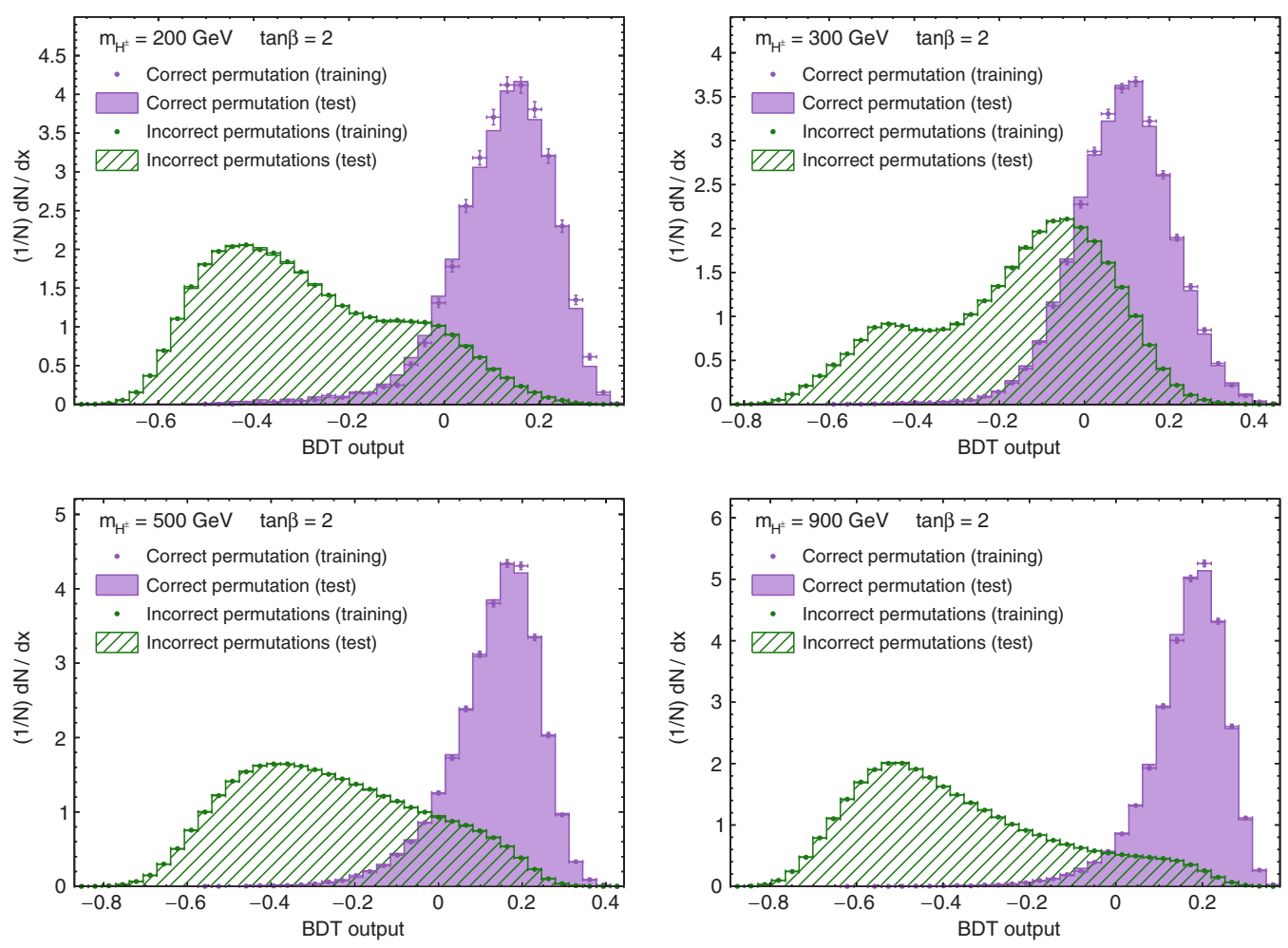

FIG. 5. Reconstruction BDT response for $m_{H^{ \pm}}=200,300,500,900 \mathrm{GeV}$ and $\tan \beta=2$.

(vi) $\Delta R\left(t_{H^{a}}, t_{c}\right), \quad \Delta \eta\left(t_{H^{a}}, t_{c}\right), \quad \Delta \phi\left(t_{H^{a}}, t_{c}\right), \quad$ where $\left(H^{a}, t_{c}\right)=\left(H^{+}, \bar{t}\right)$ or $\left(H^{-}, t\right)$;

(vii) $m\left(H^{a}\right)-m\left(b_{H}\right)$, where $a=+,-$;

(viii) $m\left(H^{+}\right)-m(\bar{t})$ and $m\left(H^{-}\right)-m(t)$;

(ix) $p_{T}^{H^{ \pm}+t_{\text {other }}}$;

(x) $m\left(H^{ \pm}, t_{\text {other }}\right)$.

Here we define $t_{H^{ \pm}}=b_{t H}+\ell_{H}, H^{ \pm}=t_{H^{ \pm}}+b_{H}$, and $t_{\text {other }}=b_{t}+\ell_{\text {other }}$, where $\ell_{H}$ is the charged lepton from the $H^{ \pm}$decay and $\ell_{\text {other }}$ is the lepton not used in defining $H^{ \pm}$.

When using the reconstruction BDT to analyze an event, we obtain the BDT output for each possible arrangement of jets and select the one with the highest value for further analysis. The BDT output distributions for correctly and incorrectly matched events is shown in Fig. 5. There is a clear separation between signal and background that improves for large $m_{H^{ \pm}}$, after falling off from $m_{H^{ \pm}}=$ 200 to $300 \mathrm{GeV}$. While these distributions are shown only for $\tan \beta=2$, the performance of the reconstruction BDT for the full range of $\tan \beta=1-60$ is shown in the second and third rows of Table I. The separation $\left\langle S^{2}\right\rangle$ is defined as

$$
\left\langle S^{2}\right\rangle=\frac{1}{2} \int \frac{\left(\hat{y}_{S}(y)-\hat{y}_{B}(y)\right)^{2}}{\hat{y}_{S}(y)+\hat{y}_{B}(y)} d y,
$$

where $y$ is the BDT response and $\hat{y}_{S}$ and $\hat{y}_{B}$ are the signal and background probability distribution functions, respectively. The performance improves with $m_{H^{ \pm}}$following a steep decline from $m_{H^{ \pm}}=200$ to $300 \mathrm{GeV}$. Also, the correct assignments are identified in a large fraction of events, bearing in mind both the large number of incorrect combinations and events in which at least one of the relevant $b$ jets is not reconstructed or chosen in the initial selection. We further note that the small variation in performance indicates that this step in the analysis is only mildly dependent on $\tan \beta$.

At low $m_{H^{ \pm}}$, we find that the most important BDT input ${ }^{6}$ is $m\left(b_{t H}, b_{H}\right)$, whereas at large $m_{H^{ \pm}}, p_{T}^{b_{H}}$ becomes the most important. The distributions for these observables are shown in Fig. 6, along with $\Delta \phi\left(b_{t H}, b_{H}\right)$. The distributions for the correct permutation vary with mass more strongly than those of the incorrect distributions, which are generally fixed for all $m_{H^{ \pm}}$. This results in a turning point around $300 \mathrm{GeV}$, where the correct and incorrect distributions are very similar. This is evident in the BDT performance metrics in Table I, which show a sharp dropoff from 200 to $300 \mathrm{GeV}$ followed by a steady increase towards larger $m_{H^{ \pm}}$.

\section{Neutrino weighting}

Once the correct jet permutation is identified, it is still necessary to determine the neutrino momenta in order to

\footnotetext{
${ }^{6}$ Here the relative importance of a observable is determined by how often it is used to split a node, weighted by the number of events in the node and the squared separation gain achieved, as defined in TMVA [53].
} 

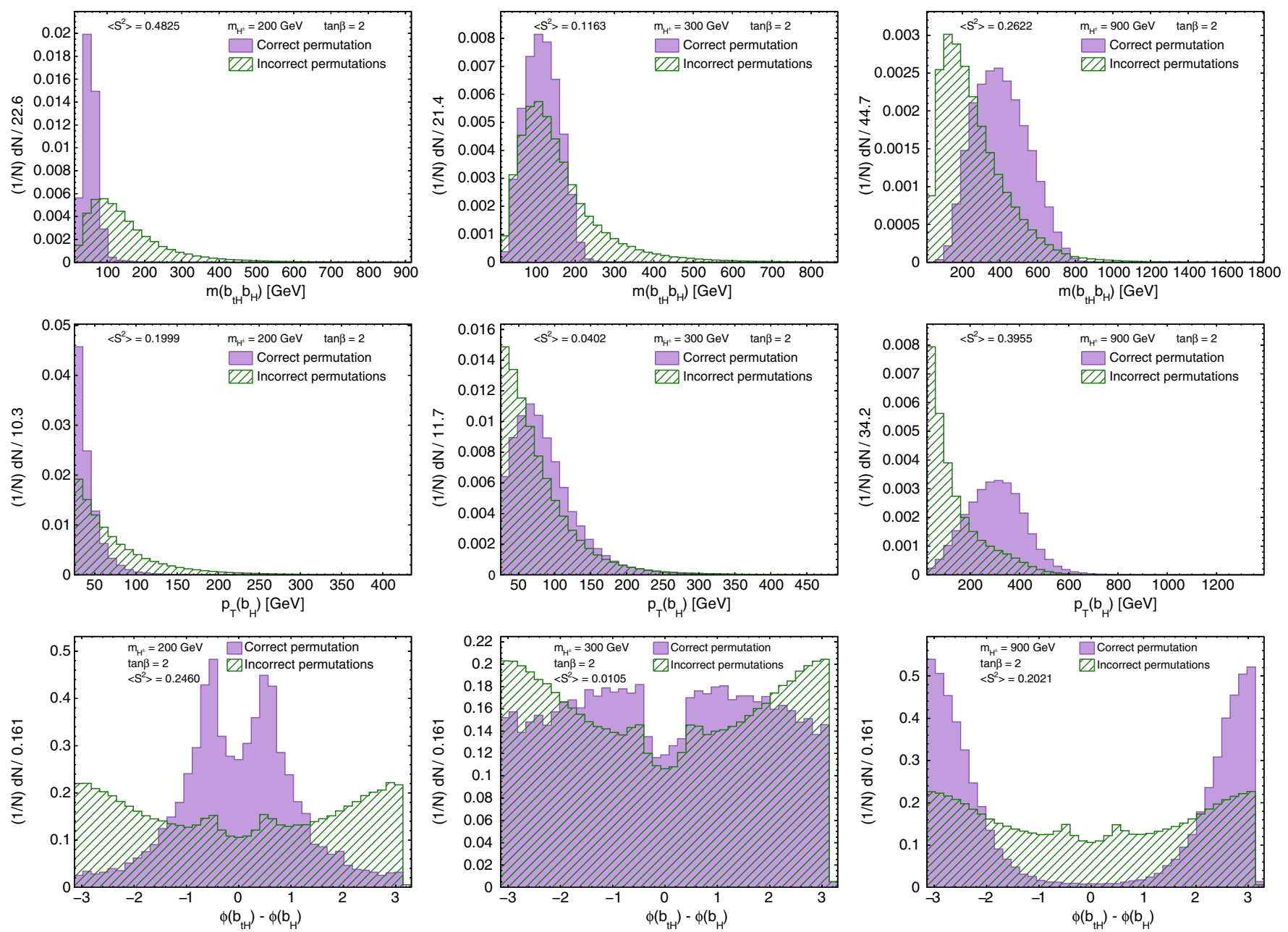

FIG. 6. Distributions of some of the most important observables in the reconstruction BDT: $m\left(b_{t H}, b_{H}\right), p_{T}^{b_{H}}$, and $\phi\left(b_{t H}\right)-\phi\left(b_{H}\right)$. Each of these observables is built entirely on charged Higgs decay products and exhibits greater separation between correct and incorrect combinations at low or high $m_{H^{ \pm}}$.

fully reconstruct the event. Since the neutrinos are reconstructed only in the form of $\mathbb{E}_{T}$, their individual momenta are unknown. Each neutrino comes from a top $\left(t \rightarrow W^{+} b\right.$, $\left.W^{+} \rightarrow \ell^{+} \nu_{\ell}\right)$ or antitop decay, which means the neutrino momenta can be constrained by the top and $W$ masses. The two $\mathbb{E}_{T}$ and four mass constraints are in principle sufficient to determine the neutrino momenta, though the quadratic nature of the mass constraints and the uncertain pairing of leptons do not provide a unique solution. To reconstruct the neutrino momenta, we follow a neutrino weighting procedure. This attempts to find the allowed pair of neutrino momenta which best reproduces the observed missing energy. Neutrino weighting is a procedure originally developed at the D0 experiment $[56,57]$ for top quark mass measurements; it has since been used in other measurements, such as the $t \bar{t}$ differential cross section at ATLAS [58]. To the best of our knowledge, it has never been used before in an analysis of the $t \bar{t} b \bar{b}$ channel. In our implementation of neutrino weighting, we sample values from a Gaussian for the pseudorapidity of the two neutrinos, $\eta_{1}$ and $\eta_{2}$, in the range $-5 \leq \eta_{i} \leq 5$. To account for variation in the invariant masses of the top quarks and increase the likelihood of finding real solutions, we also scan between 171.5 and $174.0 \mathrm{GeV}$ independently for both $m_{t}$ and $m_{\bar{t}}$. To account for jet resolution, we similarly iterate over several energies of the $b$ jets, sampling from a Gaussian. For each set of values considered, we solve for the momentum of each neutrino using

$$
\begin{aligned}
\left(p_{b}+p_{\ell}+p_{\nu}\right)^{2} & =m_{t}^{2}, \\
\left(p_{\ell}+p_{\nu}\right)^{2} & =m_{W}^{2} .
\end{aligned}
$$

This reduces to a quadratic constraint for each neutrino, producing up to four real solutions overall. Additionally, there are two possible ways to pair the leptons and $b$ jets. For each solution, we calculate a weight

$w=\exp \left(-\frac{\left(\mathbb{E}_{T}^{\mathrm{calc}}-\mathbb{E}_{T}^{\mathrm{obs}}\right)^{2}}{2 \sigma_{\phi_{T}}^{2}}\right) \exp \left(-\frac{\left(\phi^{\mathrm{calc}}-\phi^{\mathrm{obs}}\right)^{2}}{2 \sigma_{\phi}^{2}}\right)$, 

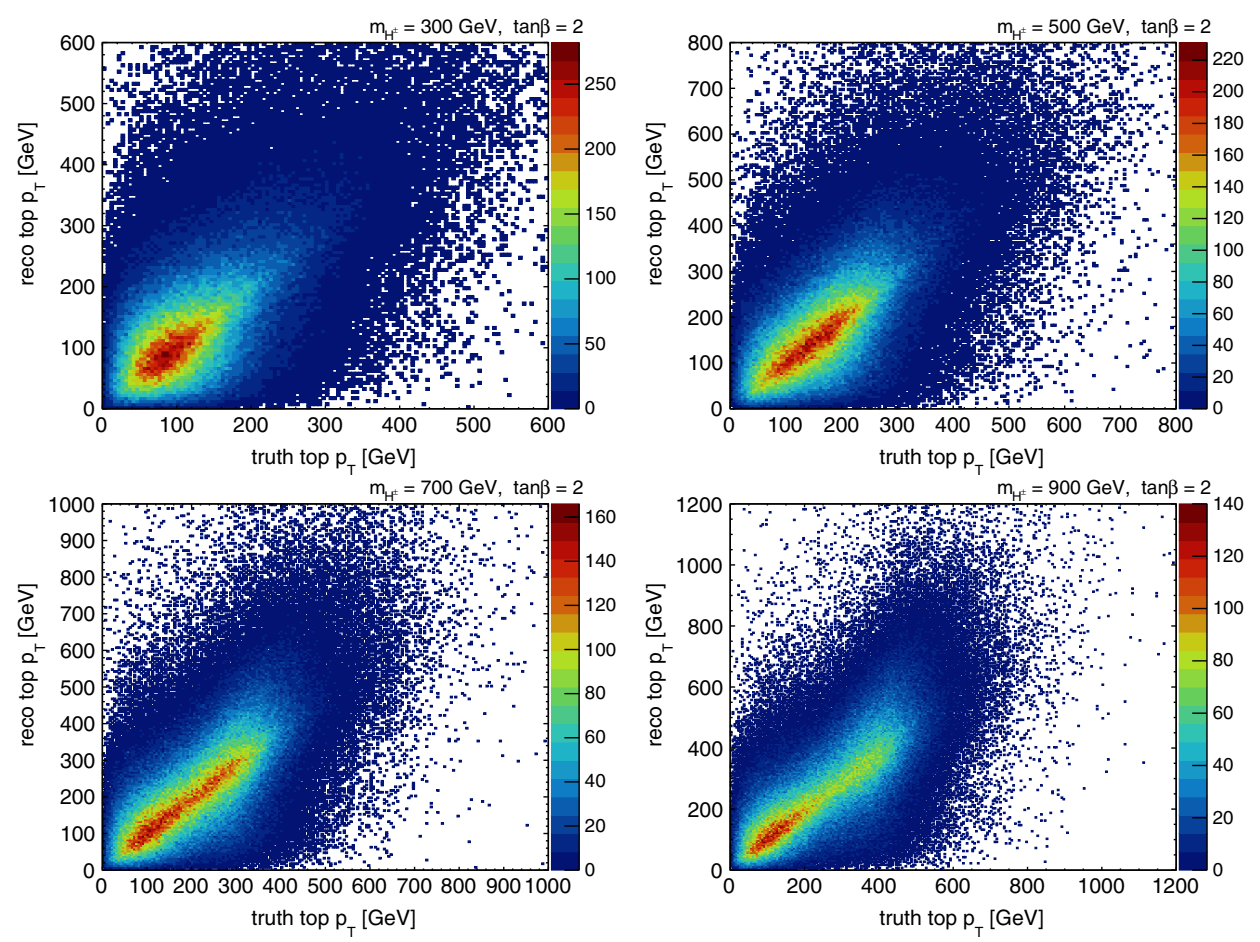

FIG. 7. Comparison of $p_{T}$ for the truth top and the reconstructed top using the reconstruction BDT and neutrino weighting procedure, for $m_{H^{ \pm}}=300,500,700,900 \mathrm{GeV}$ and $\tan \beta=2$.

where $\phi$ is the azimuthal angle of the missing energy. The resolution of $\mathbb{E}_{T}^{\mathrm{obs}}$ is given by $\sigma_{\not \not_{T}}=0.2 \mathbb{E}_{T}$, following the ATLAS resolution [59]. The resolution of $\phi^{\text {obs }}$ is given by $\sigma_{\phi}=0.05$, a fixed value based on the $\mathbb{E}_{T}$ resolution in studies from $Z$ boson events during ATLAS run 1. Out of all the combinations and solutions, we choose the one with the highest weight and take the corresponding neutrino momenta and $\ell-b$ pairings for the remainder of our analysis. If the neutrino weighting procedure is unable to find a real solution for any configuration, the event is discarded.

The performance of the neutrino weighting procedure is shown in Table I. We see that a solution is found a high percentage of the time, and the $\ell-b$ pair coming from the charged Higgs decay, which indicates the charge of the Higgs boson, is identified correctly more often than not. Since this procedure uses the $b$-jet assignments from the reconstruction BDT, to isolate the performance of neutrino weighting, we also show in Table I the fraction of events correctly reconstructing the charge of the Higgs boson when considering only events for which the $b$ jets have been correctly assigned, which we find happens for $80 \%-$ $90 \%$ of such events.

As with the reconstruction BDT, the neutrino weighting procedure sees only a small variation in performance over the range of $\tan \beta$. The fraction of events for which all $b$ jets and leptons are correctly assigned is shown in Table I. Despite seemingly low efficiencies, this is largely a reflection of the large number of possible $b$-jet permutations. The procedure performs much better than a random choice. Ultimately, we use the reconstructed neutrino momenta to reconstruct the top quarks. In Fig. 7 the reconstructed (reco) top $p_{T}$ is compared to the corresponding truth top $p_{T}$. These show strong correlations.

\section{Classification}

After the reconstruction BDT and neutrino weighting procedure, we have determined the grouping and momenta for all of the final state particles shown in Fig. 1. This allows us to reconstruct a charged Higgs mass, which can help discriminate between the signal and background, especially for large masses. However, a stronger discriminant can be constructed by taking advantage of the full kinematic information with a second BDT. This classification BDT is trained on the charged Higgs signal and the combined SM backgrounds after reconstructing the events. The classification BDT is trained on 21 observables:

(i) maximum weight from the reconstruction BDT,

(ii) $H_{T}=\sum_{i}\left|\vec{p}_{i, T}\right|$ for $i=$ all jets and leptons

(iii) centrality $=H_{T} / E$, where $E=\sum_{i} E_{i}$ for $i=$ all jets and leptons,

(iv) $m\left(b_{i}, b_{j}\right)$ and $m\left(b_{i}, l_{j}\right)$ for $i, j$ giving smallest $\Delta R$,

(v) $\min \left(m\left(b_{i}, l_{j}\right)\right)$ and $\max \left(m\left(b_{i}, l_{j}\right)\right)$,

(vi) $m\left(b_{1}, b_{2}\right), m\left(t_{H}, b_{H}\right)$,

(vii) $p_{T}^{b_{1}+b_{2}}, p_{T}^{b_{H}}, p_{T}^{b_{1}+t_{1}}, p_{T}^{t_{H}}, p_{T}^{t_{\text {other }}}$, 

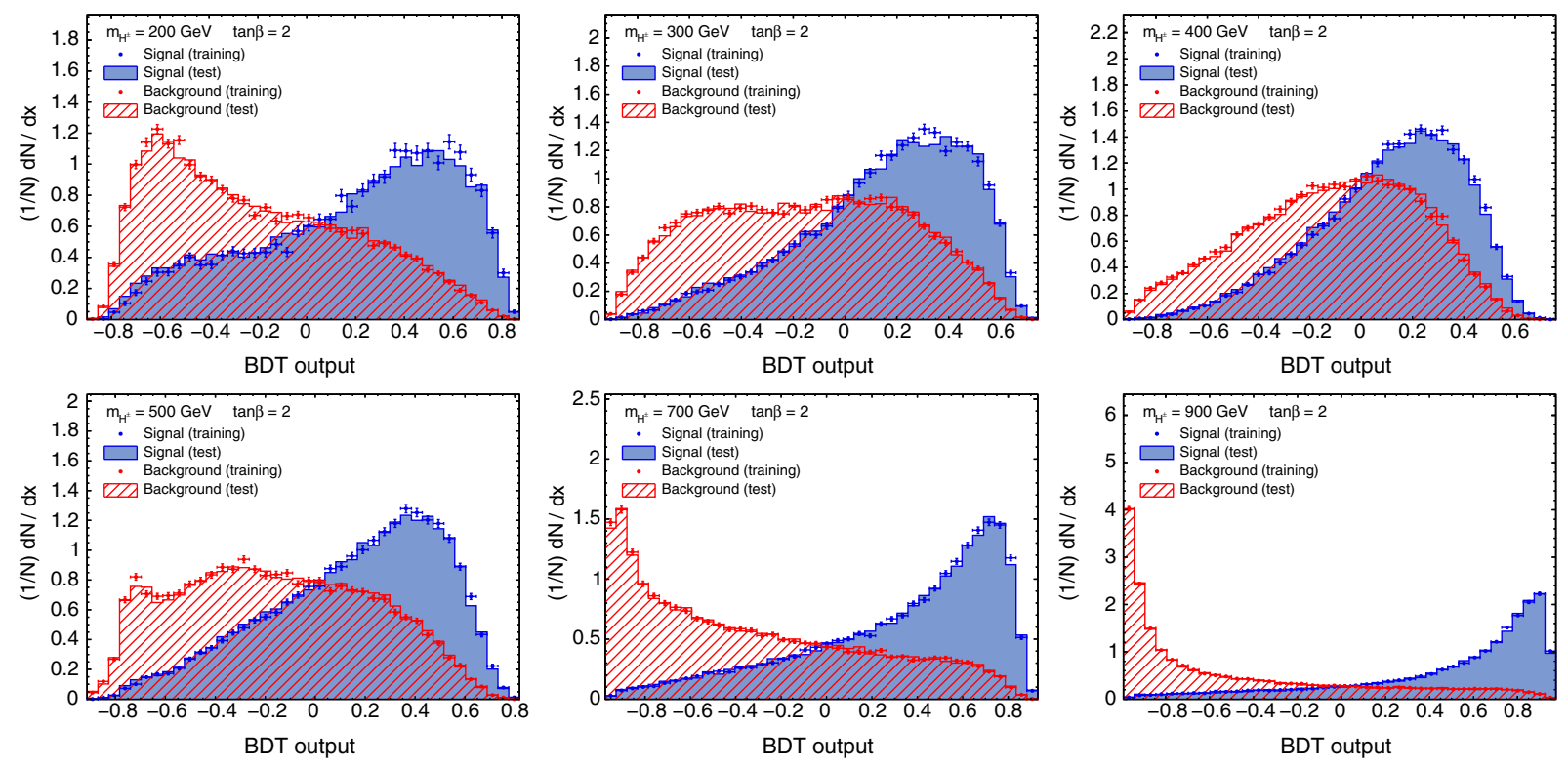

FIG. 8. Classification BDT response for $m_{H^{ \pm}}=200,300,400,500,700,900 \mathrm{GeV}$ and $\tan \beta=2$.

(viii) $\Delta R\left(b_{1}, b_{2}\right), \Delta R\left(b_{1}, t_{1}\right), \Delta R\left(t_{H}, t_{\text {non-H }}\right), \Delta R\left(t_{H}, b_{H}\right)$, $\Delta R\left(b_{t H}, b_{H}\right), \Delta R\left(b_{t}, b_{H}\right)$, and

(ix) $\cos \theta\left(l_{H}, b_{H}\right)$, the angle between $b_{H}$ and $\ell_{t H}$ in the reconstructed $H^{ \pm}$rest frame.

Here, $b_{1}\left(b_{2}\right)$ is the $b$ jet with the (second-)highest $p_{T} ; t_{1}$ is the reconstructed top (including the neutrino) with the highest $p_{T}$. In general, observables such as $m\left(j_{1}, j_{2}\right)$, the reconstruction $\mathrm{BDT}$ weight, and $m\left(t_{H}, b_{H}\right)$ contribute highly to the BDTs. The observables $p_{T}^{b_{H}}$ and $H_{T}$ become important at higher mass.

The performance of the classification BDT is shown for several charged Higgs masses in Fig. 8. While there is only a small separation between the signal and background at low $m_{H^{ \pm}}$, as $m_{H^{ \pm}}$increases the discriminating power of the BDT increases. This is also apparent in Table II. As with the reconstruction BDT, there is also an increase in separation at low $m_{H^{ \pm}}$, though the effect is smaller here. The cause of the drop in separation at 300 and $400 \mathrm{GeV}$ is because the

TABLE II. Separation $\left\langle S^{2}\right\rangle$ between the signal and background in the classification BDT.

\begin{tabular}{lccccccc}
\hline \hline & \multicolumn{7}{c}{$\tan \beta$} \\
\cline { 2 - 8 }$m_{H^{ \pm}}[\mathrm{GeV}]$ & 1 & 2 & 5 & 10 & 15 & 30 & 60 \\
\hline 200 & 0.19 & 0.19 & 0.20 & 0.22 & 0.23 & 0.23 & 0.22 \\
300 & 0.15 & 0.15 & 0.15 & 0.16 & 0.17 & 0.17 & 0.16 \\
400 & 0.10 & 0.10 & 0.10 & 0.11 & 0.12 & 0.12 & 0.11 \\
500 & 0.15 & 0.15 & 0.14 & 0.14 & 0.15 & 0.15 & 0.14 \\
600 & 0.23 & 0.24 & 0.23 & 0.21 & 0.22 & 0.22 & 0.21 \\
700 & 0.31 & 0.33 & 0.32 & 0.30 & 0.30 & 0.30 & 0.29 \\
800 & 0.40 & 0.41 & 0.40 & 0.38 & 0.38 & 0.39 & 0.36 \\
900 & 0.46 & 0.49 & 0.48 & 0.46 & 0.46 & 0.46 & 0.43 \\
1000 & 0.52 & 0.55 & 0.54 & 0.52 & 0.52 & 0.52 & 0.49 \\
\hline \hline
\end{tabular}

kinematics of the signal and background are most similar at these masses. This can be seen in the invariant mass of the reconstructed charged Higgs, for example, which is shown in Fig. 9. The classification BDT at $200 \mathrm{GeV}$ performs better largely because of the reconstruction BDT weight.

The dependence of separation on $\tan \beta$ is mild for the entire mass range. One of the observables with the largest $\tan \beta$ dependence in the classification BDT is $\cos \theta\left(l_{H}, b_{H}\right)$, shown in the Appendix.

\section{E. LHC sensitivity}

We determine the sensitivity of this analysis setup at the LHC. As a benchmark, we assume an integrated luminosity of $150 \mathrm{fb}^{-1}$, corresponding to LHC run 2 . We derive limits on the $H^{ \pm}$mediated $p p \rightarrow t \bar{t} b \bar{b}$ cross section using the $C L_{S}$ method [60]. The samples are split into a set of signal and control regions based on the number of jets and the number of $b$-tagged jets in each event. The signal regions are $\geq 4 \mathrm{j} \geq 4 b, \geq 4 \mathrm{j} 3 b$ and $3 \mathrm{j} 3 b$; the control regions are $3 \mathrm{j} 2 b$ and $4 \mathrm{j} 2 b$, used to gain a handle on the background in the fit. The derived limits are shown in Fig. 10 for several values of $\tan \beta$ and compared with the theoretical signal cross section in the MS2HDM, given by the LHC Higgs Cross Section Working Group [48-52]. Figure 11 shows that, at this luminosity, we can exclude small and large values of $\tan \beta$, for which the $H^{ \pm} t b$ coupling is the largest.

While the theoretical cross section has a strong dependence on $\tan \beta$, the expected limits on $\sigma \times \mathrm{BR}$ depend only mildly on $\tan \beta$, as shown in the Appendix. Consequently, we expect that cross-section limits set by this analysis would readily extend to other realizations of the 2HDM, as well as other models with a charged scalar coupling to third-generation quarks. 

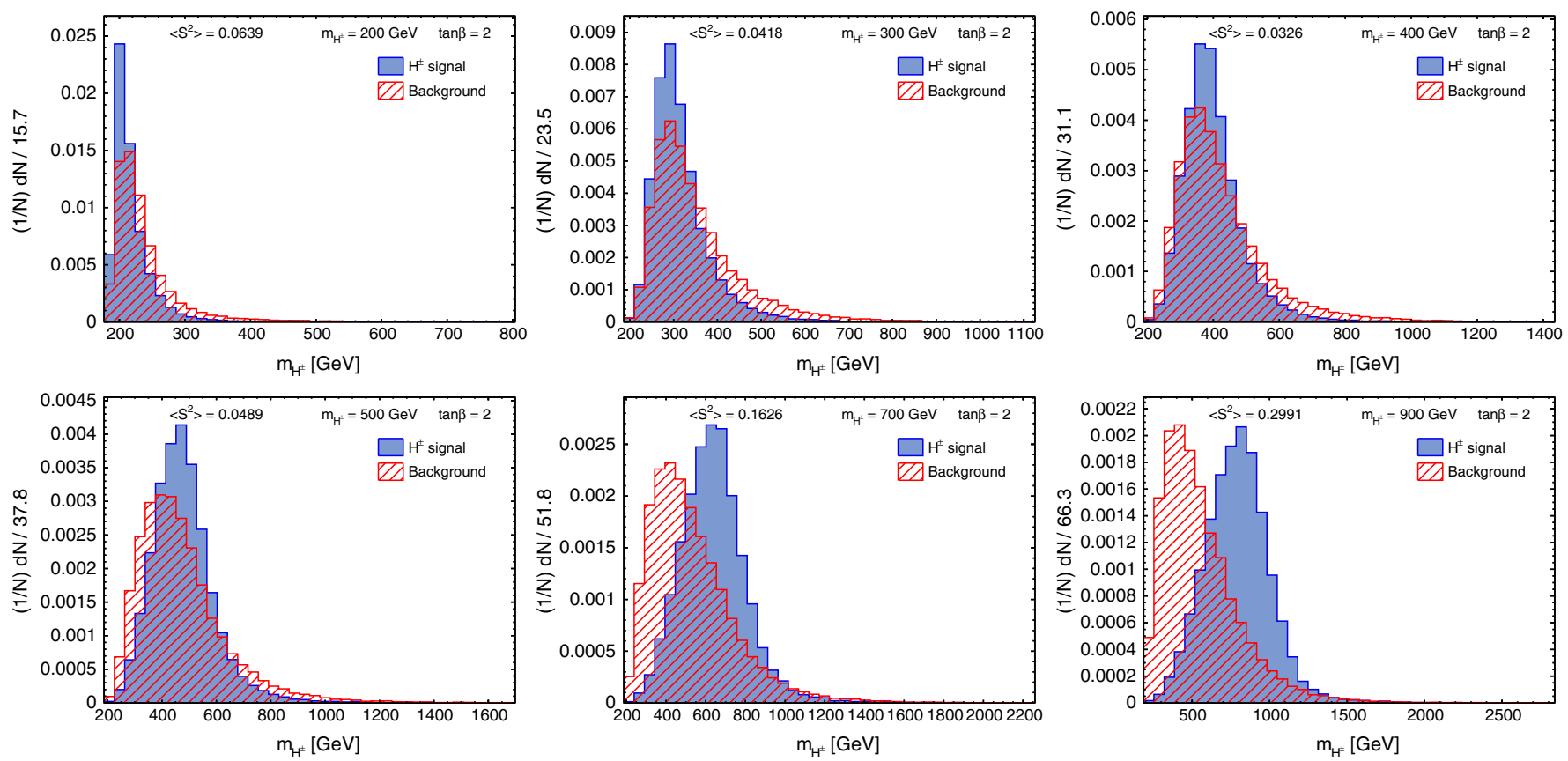

FIG. 9. Invariant mass of the reconstructed charged Higgs boson at different generated masses for $\tan \beta=2$. The charged Higgs mass is reconstructed well across all masses.

The limits are compared to those of the most recent ATLAS search in the $H^{ \pm} \rightarrow t b$ decay channel [38], which does not seek to reconstruct the charged Higgs bosons. This search combines the dilepton and lepton + jets channels. As our study is restricted to the dilepton channel, a fair comparison is made by training the classification BDT described in Ref. [38] on our MC samples. The limits obtained from this approach are then compared to the results in Fig. 10. For intermediate masses (400-600 GeV), improvements of $10 \%-15 \%$ may be seen by including the information from reconstruction. As well as improving these limits, a major benefit of the reconstruction is that it provides variables that allow the possibility, in the future, of differential cross-section measurements.
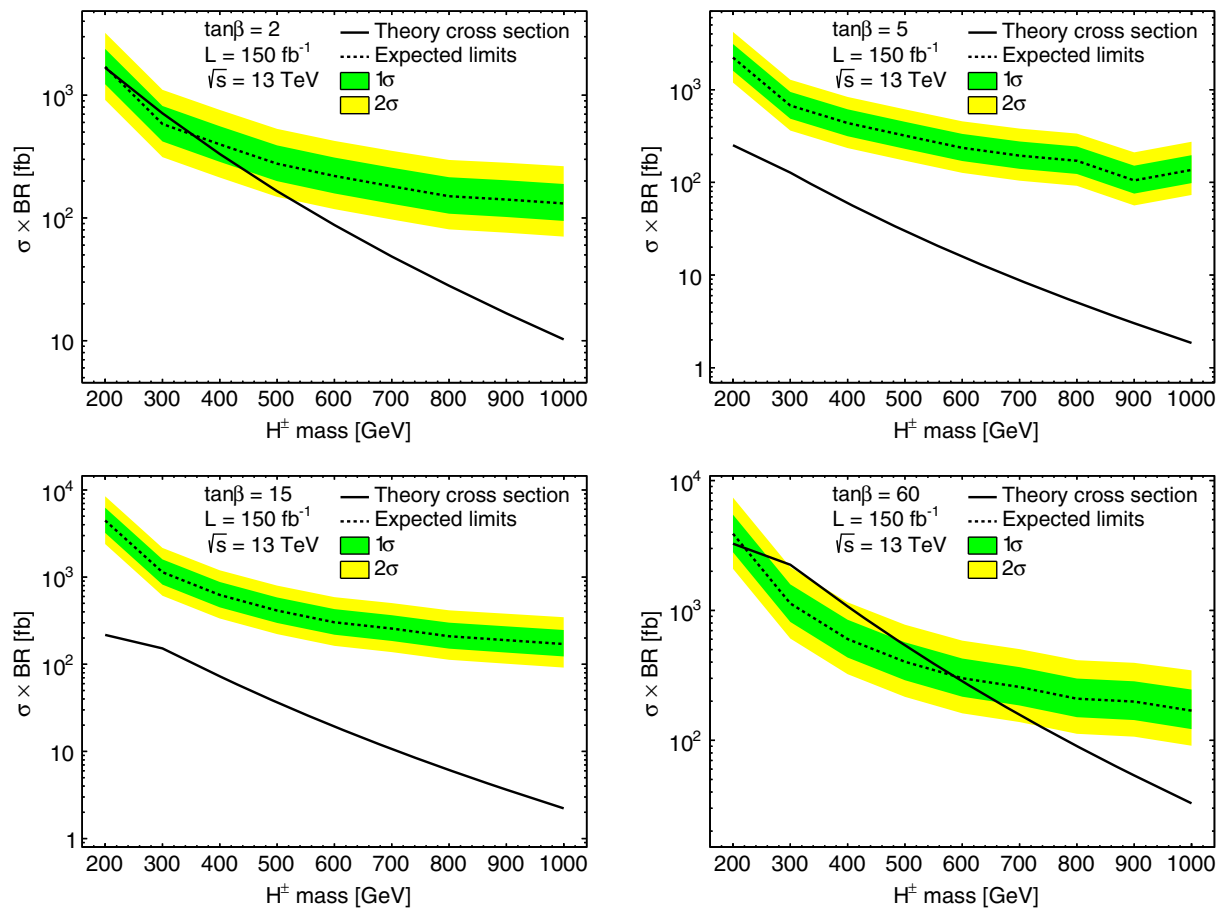

FIG. 10. Exclusion limits on $\sigma \times \mathrm{BR}$ for $\tan \beta=2,5,15,60$. 


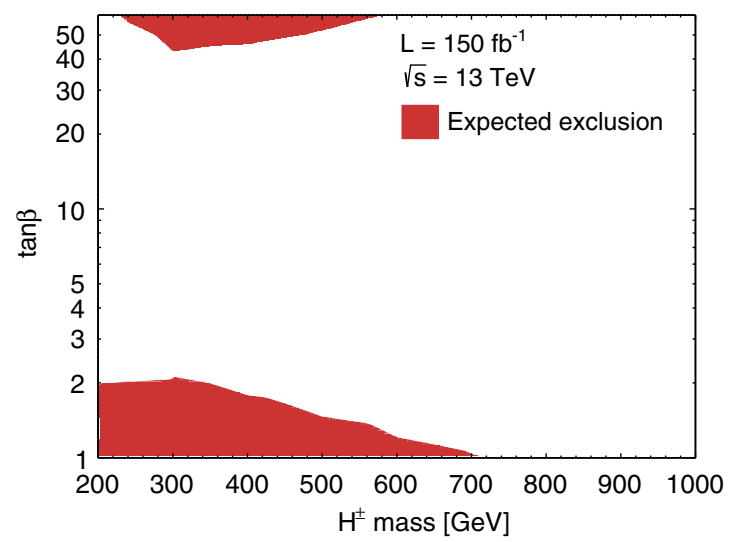

FIG. 11. Exclusion limits for the MS2HDM at the $13 \mathrm{TeV}$ LHC with an integrated luminosity of $150 \mathrm{fb}^{-1}$.

\section{CONCLUSIONS}

In this article, we have shown that the charged Higgs boson of the MS2HDM may be probed in the dileptonic $t \bar{t}$ channel at the LHC for large and small values of $\tan \beta$ and masses as large as $\sim 680 \mathrm{GeV}$. This analysis could be extended by including the single lepton and fully hadronic channels. Additionally, there exist contributions to the $t \bar{t} b \bar{b}$ channel from the two neutral MS2HDM states, $H$ and $A$, as shown in Fig. 2. Due to differences in their kinematic distributions, it is likely these neutral states would warrant a separate analysis which could be combined with this result. The neutral states could also mediate significant $4 t$ or $4 b$ cross sections, depending on the value of $\tan \beta$.
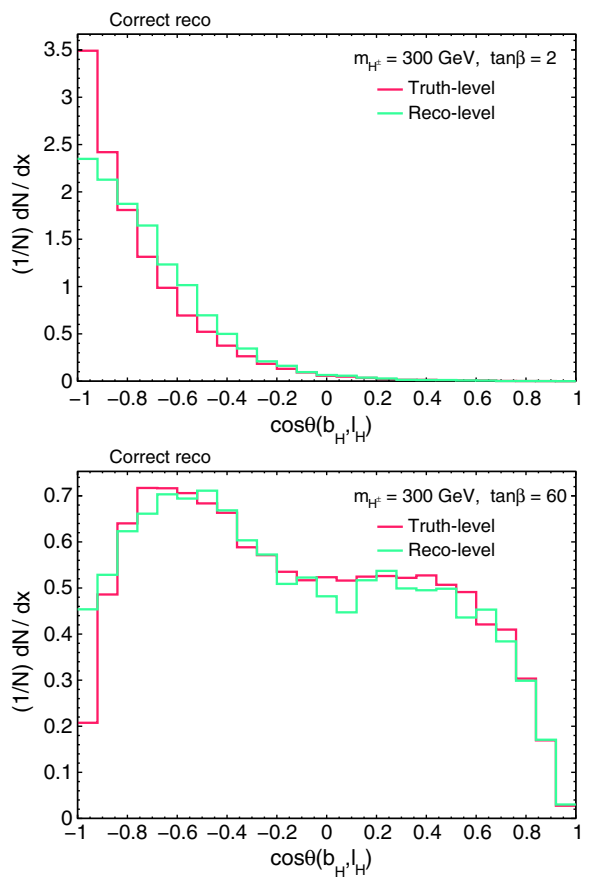

Furthermore, the analysis presented here generalizes to other realizations of the $2 \mathrm{HDM}$ and any theory containing a charged scalar with large couplings to third-generation quarks. The small variation in the final limits on $\sigma \times \mathrm{BR}$ with $\tan \beta$ suggest that they are dominated by kinematics, rather than other model parameters. This means that they can be readily applied to similar scenarios with little loss of accuracy. In conclusion, the success of the neutrino weighting procedure, which we implemented in the $t \bar{t} b \bar{b}$ channel for the first time in this article, gives us renewed impetus to apply this procedure to similar channels in the near future.

\section{ACKNOWLEDGMENTS}

The authors would like to thank Arghya Choudhury and Bhupal Dev for collaboration at the early stages of this project. The work of Emily Hanson and Yvonne Peters are supported partially by the ERC research Grant No. 335696COLORTTH, and partially by the STFC research Grant No. ST/N504178/1. Finally, the work of A. P. is supported in part by the Lancaster-Manchester-Sheffield Consortium for Fundamental Physics, under STFC research Grant No. ST/ L000520/1.

\section{APPENDIX: DEPENDENCE ON $\tan \beta$}

Figure 12 is an example observable that demonstrates the effect of $\tan \beta$ on the kinematics of the signal. Good agreement is seen in general between the truth $b$ partons and the reconstructed jets (from the reconstruction BDT),
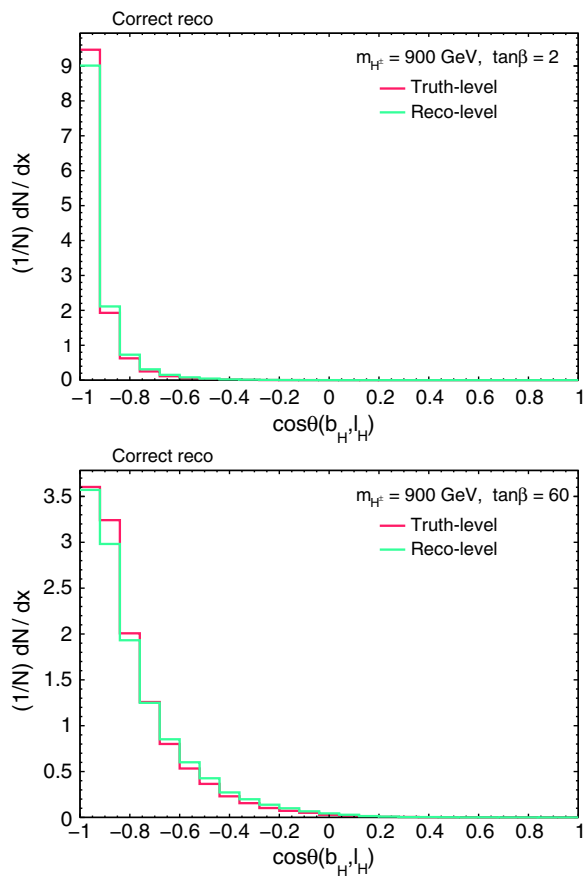

FIG. 12. The observable $\cos \theta\left(l_{H}, b_{H}\right)$ for the truth partons and for reconstructed jets at $\tan \beta=2,60$ and $m_{H^{ \pm}}=300,900 \mathrm{GeV}$, for events where the charged Higgs is correctly reconstructed. 

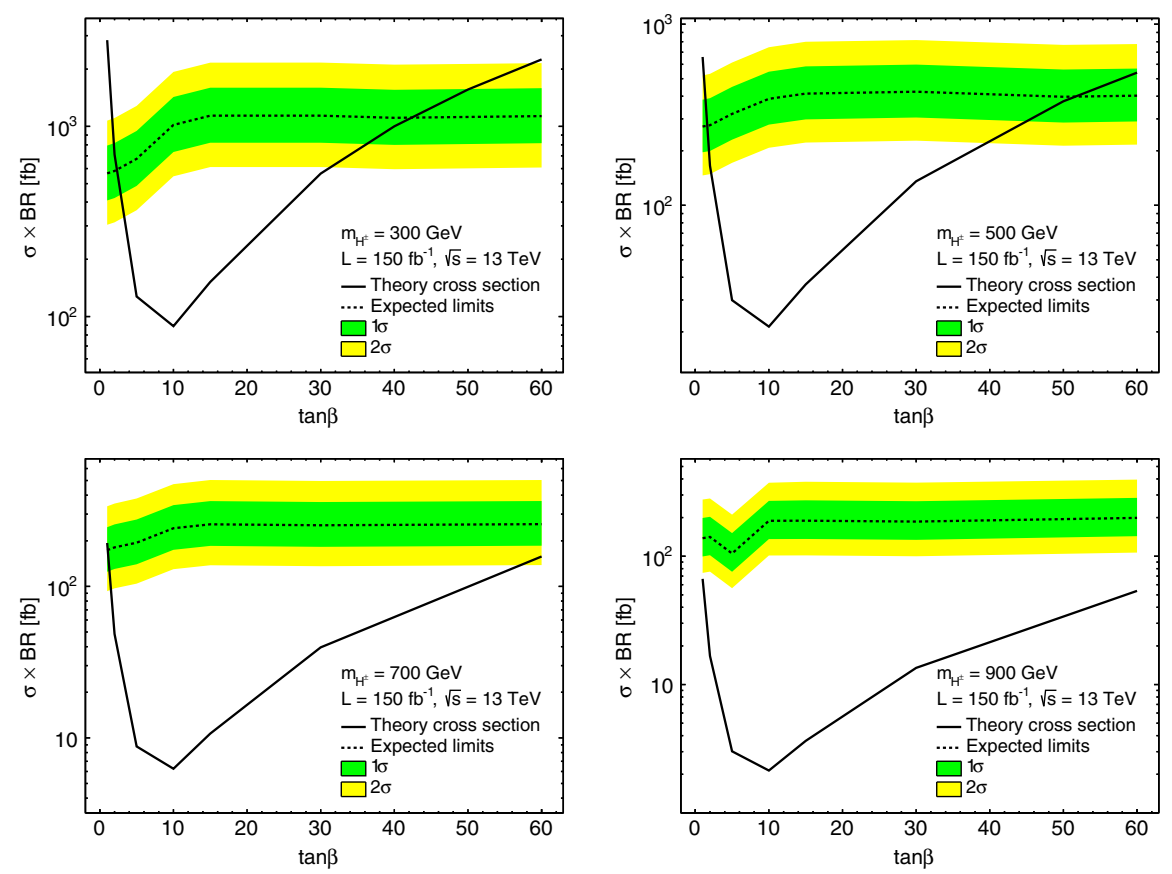

FIG. 13. Exclusion limits on $\sigma \times \mathrm{BR}$ vs $\tan \beta$ for $m_{H^{ \pm}}=300,500,700,900 \mathrm{GeV}$.

improving at higher mass where the reconstruction method has better performance. From the figure, we can see that $l_{H}$ and $b_{H}$ are generally produced back to back. At larger $\tan \beta$, the angular spread increases.
Figure 13 shows the dependence of $\tan \beta$ on the limits, shown by the dashed line, for given masses. In general, the $\tan \beta$ dependence is small. The solid line is the theoretical cross section.
[1] G. Aad et al. (ATLAS Collaboration), Phys. Lett. B 716, 1 (2012).

[2] S. Chatrchyan et al. (CMS Collaboration), Phys. Lett. B 716, 30 (2012).

[3] G. Aad et al. (ATLAS and CMS Collaborations), J. High Energy Phys. 08 (2016) 045.

[4] G. C. Branco, P. M. Ferreira, L. Lavoura, M. N. Rebelo, M. Sher, and J. P. Silva, Phys. Rep. 516, 1 (2012).

[5] H. E. Haber and G. L. Kane, Phys. Rep. 117, 75 (1985).

[6] A. Pilaftsis and C. E. M. Wagner, Nucl. Phys. B553, 3 (1999).

[7] A. Djouadi, Phys. Rep. 459, 1 (2008).

[8] J. F. Gunion and H. E. Haber, Phys. Rev. D 67, 075019 (2003).

[9] M. Carena, I. Low, N. R. Shah, and C. E. M. Wagner, J. High Energy Phys. 04 (2014) 015.

[10] P. S. B. Dev and A. Pilaftsis, J. High Energy Phys. 12 (2014) 024; 11 (2015) 147.

[11] J. Bernon, J. F. Gunion, H. E. Haber, Y. Jiang, and S. Kraml, Phys. Rev. D 92, 075004 (2015).

[12] P. M. Ferreira, R. Santos, M. Sher, and J. P. Silva, Phys. Rev. D 85, 035020 (2012).

[13] J. Bernon, J. F. Gunion, H. E. Haber, Y. Jiang, and S. Kraml, Phys. Rev. D 93, 035027 (2016).
[14] A. Pilaftsis, Phys. Rev. D 93, 075012 (2016).

[15] B. Grzadkowski, H. E. Haber, O. M. Ogreid, and P. Osland, J. High Energy Phys. 12 (2018) 056.

[16] K. Benakli, M. D. Goodsell, and S. L. Williamson, Eur. Phys. J. C 78, 658 (2018).

[17] K. Benakli, Y. Chen, and G. Lafforgue-Marmet, Eur. Phys. J. C 79, 172 (2019).

[18] N. Darvishi and A. Pilaftsis, Phys. Rev. D 99, 115014 (2019).

[19] A. G. Akeroyd et al., Eur. Phys. J. C 77, 276 (2017).

[20] A. Arbey, F. Mahmoudi, O. Stal, and T. Stefaniak, Eur. Phys. J. C 78, 182 (2018).

[21] I. F. Ginzburg and M. Krawczyk, Phys. Rev. D 72, 115013 (2005).

[22] S. L. Glashow and S. Weinberg, Phys. Rev. D 15, 1958 (1977).

[23] R. A. Battye, G. D. Brawn, and A. Pilaftsis, J. High Energy Phys. 08 (2011) 020.

[24] A. Pilaftsis, Phys. Lett. B 706, 465 (2012).

[25] M. Maniatis, A. von Manteuffel, and O. Nachtmann, Eur. Phys. J. C 57, 719 (2008).

[26] I. P. Ivanov, Phys. Rev. D 77, 015017 (2008).

[27] C. C. Nishi, Phys. Rev. D 77, 055009 (2008). 
[28] P. M. Ferreira, H. E. Haber, and J. P. Silva, Phys. Rev. D 79, 116004 (2009).

[29] P. M. Ferreira, H. E. Haber, M. Maniatis, O. Nachtmann, and J. P. Silva, Int. J. Mod. Phys. A 26, 769 (2011).

[30] G. Abbiendi et al. (LEP, DELPHI, OPAL, ALEPH, and L3 Collaborations), Eur. Phys. J. C 73, 2463 (2013).

[31] T. Aaltonen et al. (CDF Collaboration), Phys. Rev. Lett. 103, 101803 (2009).

[32] V. M. Abazov et al. (D0 Collaboration), Phys. Lett. B 682, 278 (2009).

[33] P. Gutierrez (CDF and D0 Collaborations), Proc. Sci. CHARGED2010 (2010) 004.

[34] M. Aaboud et al. (ATLAS Collaboration), J. High Energy Phys. 09 (2018) 139.

[35] V. Khachatryan et al. (CMS Collaboration), J. High Energy Phys. 11 (2015) 018.

[36] G. Aad et al. (ATLAS Collaboration), Eur. Phys. J. C 73, 2465 (2013).

[37] V. Khachatryan et al. (CMS Collaboration), J. High Energy Phys. 12 (2015) 178.

[38] M. Aaboud et al. (ATLAS Collaboration), J. High Energy Phys. 18 (2018) 85.

[39] R. Enberg, W. Klemm, S. Moretti, S. Munir, and G. Wouda, Nucl. Phys. B893, 420 (2015).

[40] J. Alwall, R. Frederix, S. Frixione, V. Hirschi, F. Maltoni, O. Mattelaer, H. S. Shao, T. Stelzer, P. Torrielli, and M. Zaro, J. High Energy Phys. 07 (2014) 079.

[41] M. Misiak and M. Steinhauser, Eur. Phys. J. C 77, 201 (2017).

[42] M. Guchait and A. H. Vijay, Phys. Rev. D 98, 115028 (2018).

[43] T. Sjöstrand, S. Mrenna, and P. Z. Skands, J. High Energy Phys. 05 (2006) 026.

[44] M. Cacciari, G. P. Salam, and G. Soyez, Eur. Phys. J. C 72, 1896 (2012).
[45] J. de Favereau, C. Delaere, P. Demin, A. Giammanco, V. Lematre, A. Mertens, and M. Selvaggi (DELPHES 3 Collaboration), J. High Energy Phys. 02 (2014) 057.

[46] D. Eriksson, J. Rathsman, and O. Stål, Comput. Phys. Commun. 181, 189 (2010).

[47] M. L. Mangano, M. Moretti and R. Pittau, Nucl. Phys. B 632, 343 (2002).

[48] C. Degrande, M. Ubiali, M. Wiesemann, and M. Zaro, J. High Energy Phys. 10 (2015) 145.

[49] M. Flechl, R. Klees, M. Kramer, M. Spira, and M. Ubiali, Phys. Rev. D 91, 075015 (2015).

[50] D. de Florian et al. (LHC Higgs Cross Section Working Group), arXiv:1610.07922.

[51] S. Dittmaier, M. Krämer, M. Spira, and M. Walser, Phys. Rev. D 83, 055005 (2011).

[52] E. L. Berger, T. Han, J. Jiang, and T. Plehn, Phys. Rev. D 71, 115012 (2005).

[53] A. Hocker et al., Proc. Sci., ACAT2007 (2007) 040.

[54] Y. Freund and R. E. Schapire, Experiments with a new boosting algorithm (Morgan Kaufmann, 1996).

[55] Y. Freund and R. E. Schapire, J. Comput. Syst. Sci. 55, 119 (1997).

[56] B. Abbott et al. (D0 Collaboration), Phys. Rev. Lett. 80, 2063 (1998).

[57] B. Abbott et al. (D0 Collaboration), Phys. Rev. D 60, 052001 (1999).

[58] M. Aaboud et al. (ATLAS Collaboration), Eur. Phys. J. C 77, 292 (2017).

[59] ATLAS Collaboration, Performance of missing transverse momentum reconstruction for the ATLAS detector in the first proton-proton collisions at $\sqrt{s}=13 \mathrm{TeV}$, Technical Report No. ATL-PHYS-PUB-2015-027, CERN, Geneva, 2015.

[60] A. L. Read, J. Phys. G 28, 2693 (2002). 